\title{
ABSTRACT \\ ON HYPERBOLIC POLYNOMIALS WITH FOUR-TERM RECURRENCE AND LINEAR COEFFICIENTS
}

For any real numbers $a, b$, and $c$, we form the sequence of polynomials $\left\{P_{n}(z)\right\}_{n=0}^{\infty}$ satisfying the four-term recurrence

$$
P_{n}(z)=-a z P_{n-1}(z)-b P_{n-2}(z)-c z P_{n-3}(z), n \in \mathbb{N}
$$

with the initial conditions $P_{0}(z)=1$ and $P_{-n}(z)=0$. We find necessary and sufficient conditions on $a, b$, and $c$ under which the zeros of $P_{n}(z)$ are real for all $n$, and provide an explicit real interval on which $\bigcup_{n=0}^{\infty} \mathcal{Z}\left(P_{n}\right)$ is dense, where $\mathcal{Z}\left(P_{n}\right)$ is the set of zeros of $P_{n}(z)$.

Richard Adams

December 2018 

ON HYPERBOLIC POLYNOMIALS WITH FOUR-TERM RECURRENCE AND LINEAR COEFFICIENTS

by

Richard Adams

\author{
A thesis \\ submitted in partial \\ fulfillment of the requirements for the degree of \\ Master of Arts in Mathematics \\ in the College of Science and Mathematics \\ California State University, Fresno
}

December 2018 


\section{APPROVED}

For the Department of Mathematics:

We, the undersigned, certify that the thesis of the following student meets the required standards of scholarship, format, and style of the university and the student's graduate degree program for the awarding of the master's degree.

Richard Adams

Thesis Author

Khang Tran (Chair) Mathematics

Tamás Forgács

Mathematics

Oscar Vega Mathematics

For the University Graduate Committee:

Dean, Division of Graduate Studies 


\section{AUTHORIZATION FOR REPRODUCTION OF MASTER'S THESIS}

I grant permission for the reproduction of this thesis in part or in its entirety without further authorization from me, on the condition that the person or agency requesting reproduction absorbs the cost and provides proper acknowledgment of authorship.

$\mathrm{X}$ Permission to reproduce this thesis in part or in its entirety must be obtained from me.

Signature of thesis author: 


\section{ACKNOWLEDGMENTS}

I would like to thank my advisor, Dr. Khang Tran, for giving me the tools and guidance needed to be successful at the graduate level; his brilliant methods and patient demeanor were indispensable. I would like to thank my committee members for their helpful constructive criticism of my work and for always being available for questions. I would like to thank my undergraduate research advisor, Dr. Oscar Vega, for showing me how rewarding mathematics research can be and inspiring me to pursue graduate school. I would also like to thank my parents for instilling in me from a young age the desire to learn and question everything. 
TABLE OF CONTENTS

Page

LIST OF FIGURES . . . . . . . . . . . . . . . . . . vi

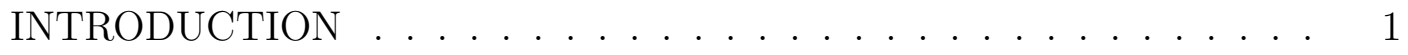

CHEBYSHEV POLYNOMIALS OF THE SECOND KIND . . . . . . 5

GENERATING FUNCTIONS . . . . . . . . . . . . . . . . 9

THE CAUCHY DIFFERENTIATION FORMULA . . . . . . . . 15

APPLICATIONS OF GENERATING FUNCTIONS . . . . . . . . . . 21

THE CUBIC DENOMINATOR . . . . . . . . . . . . . . . . 31

MAIN RESULT . . . . . . . . . . . . . . . . . . . . . 41

NECESSARY CONDITION FOR THE REALITY OF ZEROS $\ldots . .71$

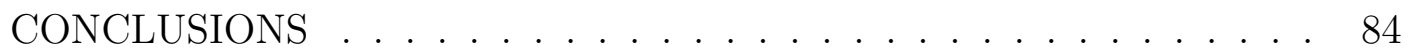

REFERENCES .......................... 86 


\section{LIST OF FIGURES}

Page

Figure 1. Zeros of $Q_{100}(z): A(z)=1, B(z)=z, C(z)=z$. . . . 12

Figure 2. Zeros of $Q_{100}(z): A(z)=1, B(z)=1, C(z)=z^{2} \ldots 13$

Figure 3. Zeros of $Q_{100}(z): A(z)=1, B(z)=z^{2}, C(z)=z^{3} . \ldots 13$

Figure 4. Zeros of $Q_{100}(z): A(z)=1, B(z)=-z^{2}+z+10, C(z)=z^{5} \cdot 14$

Figure 5. Zeros of $H_{100}(z): A(z)=1, B(z)=z, C(z)=0 \ldots 24$

Figure 6. Zeros of $K_{100}(z): A(z)=1, B(z)=0, C(z)=z . \ldots 31$ 


\section{INTRODUCTION}

Finding the zeros, or zero set, of a function is an important aspect of many branches of mathematics. For example, one of the biggest open problems of our time is to determine whether the zeros of the Riemann Zeta function all lie on the line $\operatorname{Re}(s)=1 / 2, s \in \mathbb{C}$. Of particular interest are the zero distributions of recursively defined polynomial sequences. One such type of polynomial sequence that has been widely studied since the early 19th century is called an orthogonal polynomial sequence.

Definition 1. A sequence of polynomials $\left\{p_{n}(x)\right\}_{n=0}^{\infty}$ with $\operatorname{deg}\left(p_{n}(x)\right)=n$ for each $n$ is called orthogonal with respect to some weight function $w(x)$ on the interval $(a, b)$ if

$$
\int_{a}^{b} p_{n}(x) p_{m}(x) w(x) d x=c_{n} \delta_{n m} \text { with } \delta_{n m}=\left\{\begin{array}{c}
0 \text { if } n \neq m \\
1 \text { if } n=m
\end{array}\right.
$$

Orthogonal polynomial sequences have many interesting properties and applications which can be found in $[1,6]$, such as the fact that they can be defined by a three-term recurrence relation and the zeros of any polynomial in the sequence are real and lie on the interval $(a, b)$.

Definition 2. A polynomial is said to be hyperbolic if all of its zeros are real.

Three-term polynomial recurrences have been studied extensively, but not much is known about four-term recurrences. In this thesis, we will study four-term recurrence relations defining a sequence polynomials $\left\{Q_{n}(z)\right\}_{n=0}^{\infty}$ 
satisfying

$$
Q_{n}(z)+A(z) Q_{n-1}(z)+B(z) Q_{n-2}(z)+C(z) Q_{n-3}(z)=0
$$

where $Q_{0}(z)=1, Q_{-n}(z)=0$, and $A(z), B(z)$, and $C(z)$ are some linear complex-valued polynomials with real coefficients. For recent work on the four-term recurrence, see $[4,9,10,11]$. Next we present the main theorem of this thesis.

Theorem 3. The zeros of the sequence $\left\{P_{n}(z)\right\}_{n=0}^{\infty}$ satisfying the recurrence relation

$$
P_{n}(z)+a z P_{n-1}(z)+b P_{n-2}(z)+c z P_{n-3}(z)=0, a, b, c \in \mathbb{R} \backslash\{0\},
$$

where $P_{0}(z)=1$ and $P_{-n}(z)=0$, are real if and only if $b>0$ and $\frac{c}{a b}:=\alpha \leq \frac{1}{9}$, in which case they lie on the interval $(-\lambda, \lambda)$, where

$$
\lambda:=\frac{4}{\left(\frac{3 \alpha+1+\sqrt{9 \alpha^{2}-10 \alpha+1}}{-5 \alpha+1+\sqrt{9 \alpha^{2}-10 \alpha+1}}\right)^{\frac{3}{2}}\left(-5 \alpha+1+\sqrt{9 \alpha^{2}-10 \alpha+1}\right)}
$$

Furthermore, the set of zeros of $P_{n}(z)$ is dense on $(-\lambda, \lambda)$ as $n \rightarrow \infty$.

The outline of this thesis is as follows. In the next section we introduce a well-known orthogonal polynomial sequence and demonstrate how to prove that each polynomial in the sequence is hyperbolic. In the third section we show how a generating function can be defined for any finite recurrence relation, which is then applied in the fifth section as a more convenient way of describing recurrence relations. In the fourth section we apply the Cauchy 
Differentiation Formula to rewrite generating functions in a more useful way. In the sixth section we explore the four-term recurrence and apply what we learned in the previous sections to prove a particular case. We state the main theorem in the seventh section in terms of its generating relation, and prove the sufficiency part of the theorem. Finally, in the eighth section, we prove the necessary condition of the theorem using a result by Sokal [8]. Next we state a well-known result in topology which will be useful throughout this thesis.

Theorem 4. [7] Let $X$ and $Y$ be topological spaces; let $f: X \rightarrow Y$. Then the following are equivalent:

1. $f$ is continuous

2. For every subset $A$ of $X$, one has $f(\bar{A}) \subset \overline{f(A)}$.

3. For every closed set $B$ of $Y$, the set $f^{-1}(B)$ is closed in $X$.

4. For each $x \in X$ and each neighborhood $V$ of $f(x)$, there is a neighborhood $U$ of $x$ such that $f(U) \subset V$.

For the purposes of this thesis, Theorem 4 can be interpreted as saying that the continuous image of a dense set is dense.

Remark 5. There are two things to keep in mind for this thesis.

1. We will be defining many functions of $\theta$, such as $\tau(\theta)$. For simplicity of notation, after the functions are initially defined we supress the parameter $\theta$ and write $\tau:=\tau(\theta)$. 
2. There are four main polynomial sequences considered in this thesis: $\left\{U_{n}(z)\right\}_{n=0}^{\infty},\left\{H_{n}(z)\right\}_{n=0}^{\infty},\left\{K_{n}(z)\right\}_{n=0}^{\infty}$, and $\left\{P_{n}(z)\right\}_{n=0}^{\infty}$. For any other generic polynomial sequence, the notation $\left\{Q_{n}(z)\right\}_{n=0}^{\infty}$ will be used. 


\section{CHEBYSHEV POLYNOMIALS OF THE SECOND KIND}

A classical sequence of hyperbolic polynomials is the sequence of Chebyshev polynomials of the second kind. We will illustrate our method of proving the hyperbolicity of more complicated recurrences using this sequence as a simple example.

Definition 6. The Chebyshev polynomials of the second kind, denoted $U_{n}(z)$, are complex-valued polynomials with real coefficients defined recursively by

$$
\begin{aligned}
& U_{0}(z)=1 \\
& U_{1}(z)=2 z \\
& U_{n}(z)=2 z U_{n-1}(z)-U_{n-2}(z), n \geq 2 .
\end{aligned}
$$

Example 7. Using the recursive definition, we calculate the Chebyshev polynomials up to $U_{5}(z)$.

$$
\begin{aligned}
U_{2}(z) & =2 z U_{1}(z)-U_{0}(z)=2 z(2 z)-1=4 z^{2}-1 . \\
U_{3}(z) & =2 z U_{2}(z)-U_{1}(z)=2 z\left(4 z^{2}-1\right)-2 z=8 z^{3}-4 z . \\
U_{4}(z) & =2 z U_{3}(z)-U_{2}(z)=2 z\left(8 z^{3}-4 z\right)-\left(4 z^{2}-1\right)=16 z^{4}-12 z^{2}+1 . \\
U_{5}(z) & =2 z U_{4}(z)-U_{3}(z) \\
& =2 z\left(16 z^{4}-12 z^{2}+1\right)-\left(8 z^{3}-4 z\right) \\
& =32 z^{5}-32 z^{3}+6 z .
\end{aligned}
$$

We notice that all the zeros of each polynomial are real, but we wish to determine whether $U_{n}(z)$ is hyperbolic for all $n$. 
Proposition 8. All zeros of Chebyshev polynomials of the second kind are real and lie on the interval $(-1,1)$, where they are dense as $n \rightarrow \infty$.

Proof. For $\theta \in(0, \pi)$, consider the sequence

$$
g_{U_{n}}(\theta):=\frac{\sin (n+1) \theta}{\sin (\theta)}, n \in \mathbb{N}
$$

and define the function

$$
z_{U}(\theta):=\cos \theta
$$

We will show that $g_{U_{n}}(\theta)=U_{n}(\cos \theta)$ by proving that the two sequences satisfy the same recurrence relation and initial conditions.

For $n=0$, we have $g_{U_{0}}=\frac{\sin \theta}{\sin \theta}=1$.

For $n=1$, we have $g_{U_{1}}=\frac{\sin 2 \theta}{\sin \theta}=2 \cos \theta$.

Since $z_{U}=\cos \theta, g_{U_{0}}$ and $g_{U_{1}}$ correspond to $U_{0}(z)$ and $U_{1}(z)$, respectively.

Observe that for $n \geq 2$,

$$
g_{U_{n}}=\frac{\sin (n+1) \theta}{\sin (\theta)}=\frac{\sin (n \theta+\theta)}{\sin (\theta)} .
$$

Applying the trigonometric identity $\sin (x+y)=\sin x \cos y+\cos x \sin y$, Equation (3) becomes

$$
g_{U_{n}}=\frac{\sin (n \theta) \cos (\theta)+\cos (n \theta) \sin (\theta)}{\sin (\theta)} .
$$


Adding and subtracting $\sin (n \theta) \cos \theta$, and then applying the trigonometric identity $\sin (x-y)=\sin x \cos y-\cos x \sin y$, Equation (4) is equivalent to

$$
\begin{aligned}
g_{U_{n}} & =\frac{2 \cos (\theta) \sin (n \theta)}{\sin (\theta)}-\frac{\sin (n \theta) \cos (\theta)-\cos (n \theta) \sin (\theta)}{\sin (\theta)} \\
& =\frac{2 \cos (\theta) \sin (n \theta)}{\sin (\theta)}-\frac{\sin (n \theta-\theta)}{\sin (\theta)} \\
& =\frac{2 \cos (\theta) \sin (n \theta)}{\sin (\theta)}-\frac{\sin ((n-1) \theta)}{\sin (\theta)} \\
& =2 \cos (\theta) g_{U_{n-1}}-g_{U_{n-2}} \\
& =2 z_{U} g_{U_{n-1}}-g_{U_{n-2}} .
\end{aligned}
$$

Since $\left\{g_{U_{n}}\right\}_{n=0}^{\infty}$ and $\left\{U_{n}(z)\right\}_{n=0}^{\infty}$ satisfy the same initial conditions and the same recurrence relation, they must be the same sequence. We find the zeros of $g_{U_{n}}$ to be

$$
\theta=\frac{k \pi}{n+1}, k \in \mathbb{Z}
$$

and since $\theta \in(0, \pi), k \in\{1,2, \ldots, n\}$. Hence there are $n$ zeros of $g_{U_{n}}$ on $(0, \pi)$, and they occur only when

$$
z_{U}=\cos \theta=\cos \left(\frac{k \pi}{n+1}\right) \in(-1,1), 1 \leq k \leq n
$$

Note that $z_{U}$ is monotone on $(0, \pi)$, so each distinct solution of $g_{U_{n}}=0$ corresponds to a distinct solution of $U_{n}(z)=0$ by $z_{U}$. From Definition 8 , it is clear that $\operatorname{deg}\left(U_{n}(z)\right)=n$ for all $n$, and since we have found $n$ zeros of $g_{U_{n}}$ on $(0, \pi)$, we have by the Fundamental Theorem of Algebra that all the zeros of $U_{n}(z)$ are real and lie on the interval $(-1,1)$. The density of zeros comes from the fact that the interval $(0, \pi)$ is partitioned into $n+1$ subintervals, with 
each interval endpoint (except 0 and $\pi$ ) being a solution of $g_{U_{n}}=0$. Hence as $n \rightarrow \infty$, any arbitrarily small subinterval of $(0, \pi)$ will contain a zero of $g_{U_{n}}$. So the set of zeros of $g_{U_{n}}$ is dense on $(0, \pi)$ as $n \rightarrow \infty$. Since $z_{U}:(0, \pi) \rightarrow(-1,1)$ is continuous and bijective, we have by Theorem 4 that the set of zeros of $U_{n}(z)$ is dense on $(-1,1)$ as $n \rightarrow \infty$. 


\section{GENERATING FUNCTIONS}

Another way of representing the sequence $\left\{U_{n}(z)\right\}_{n=0}^{\infty}$ is in terms of its generating function. Recall the geometric power series

$$
\frac{1}{1-t}=\sum_{n=0}^{\infty} t^{n},|t|<1
$$

To prove this formula, one can easily show that

$$
(1-t) \sum_{n=0}^{\infty} t^{n}=1
$$

by expanding the product of the finite sum and canceling terms as follows:

$$
(1-t) \sum_{n=0}^{N} t^{n}=(1-t)\left(1+t+t^{2}+\cdots+t^{N}\right)=1-t^{N+1}
$$

which approaches 1 as $N \rightarrow \infty$ since $|t|<1$. We demonstrate from an example that the coefficients of the Maclaurin series of a rational function satisfy a finite recurrence.

Example 9. Consider the Maclaurin series of the function

$$
\frac{1}{1-3 t+2 t^{2}}=\sum_{n=0}^{\infty} a_{n} t^{n}, a_{n} \in \mathbb{C}
$$

We find a recurrence relation for the sequence $\left\{a_{n}\right\}_{n=0}^{\infty}$. Observe

$$
\begin{aligned}
1 & =\left(1-3 t+2 t^{2}\right) \sum_{n=0}^{\infty} a_{n} t^{n} \\
& =\sum_{n=0}^{\infty} a_{n} t^{n}-3 t \sum_{n=0}^{\infty} a_{n} t^{n}+2 t^{2} \sum_{n=0}^{\infty} a_{n} t^{n} .
\end{aligned}
$$


After collecting powers of $t$, we have

$$
1=a_{0}+\left(a_{1}-3 a_{0}\right) t+\sum_{n=2}^{\infty}\left(a_{n}-3 a_{n-1}+2 a_{n-2}\right) t^{n}
$$

which, by matching coefficients gives

$$
\begin{aligned}
a_{0} & =1, \\
a_{1}-3 a_{0} & =0, \quad \text { and } \\
a_{n}-3 a_{n-1}+2 a_{n-2} & =0, \quad n \geq 2 .
\end{aligned}
$$

Hence we get get the recurrence

$$
a_{n}-3 a_{n-1}+2 a_{n-2}=0
$$

with $a_{0}=1$ and $a_{-n}=0$, or equivalently,

$$
a_{n}=3 a_{n-1}-2 a_{n-2},
$$

with $a_{0}=1$ and $a_{-n}=0$.

We generalize this process for a finite recurrence in the following lemma.

Lemma 10. Let $A_{1}(z), A_{2}(z), \ldots, A_{m}(z)$ be polynomials in $z$. For a fixed $z$, the sequence $\left\{Q_{n}(z)\right\}_{n=0}^{\infty}$ generated by

$$
\sum_{n=0}^{\infty} Q_{n}(z) t^{n}=\frac{1}{1+A_{1}(z) t+A_{2}(z) t^{2}+\cdots+A_{m}(z) t^{m}}
$$


satisfies the recurrence

$$
Q_{n}(z)=-\sum_{k=1}^{m} A_{k}(z) H_{n-k}
$$

with initial conditions $Q_{0}(z)=1$ and $Q_{-n}=0$ for all $n$.

Proof. Note that for small $|t|$ and fixed $z$ the series $\sum_{n=0}^{\infty} Q_{n}(z) t^{n}$ converges absolutely. Multiplying both sides of Equation (5) by the denominator gives

$$
1=\left(1+A_{1}(z) t+A_{2}(z) t^{2}+\cdots+A_{m}(z) t^{m}\right) \sum_{n=0}^{\infty} H_{n}(z) t^{n} .
$$

Because of the absolute convergence, we may distribute, rearrange, and collect powers of $t$ to obtain

$$
\begin{aligned}
1 & =H_{0}(z) \\
& +\left(H_{1}(z)+A_{1}(z) H_{0}(z)\right) t \\
& +\left(H_{2}(z)+A_{1}(z) H_{1}(z)+A_{2}(z) H_{0}(z)\right) t^{2} \\
& +\left(H_{3}(z)+A_{1}(z) H_{2}(z)+A_{2}(z) H_{1}(z)+A_{3}(z) H_{0}(z)\right) t^{3} \\
& \vdots \\
& +\left(H_{n}(z)+A_{1}(z) H_{n-1}(z)+A_{2}(z) H_{n-2}(z)+\cdots+A_{n}(z) H_{n-m}(z)\right) t^{n}
\end{aligned}
$$$$
\vdots
$$

which, by matching coefficients gives $H_{0}(z)=1$ and

$$
H_{n}(z)=-A_{1}(z) H_{n-1}(z)-A_{2}(z) H_{n-2}(z)-\cdots-A_{m}(z) H_{n-m},
$$


where $H_{-n}=0$.

By Lemma 10, we see that the sequence of Chebyshev polynomials of the second kind can be defined using the generating relation

$$
\sum_{n=0}^{\infty} U_{n}(z) t^{n}=\frac{1}{1-2 z t+t^{2}}
$$

In this thesis, we study the zeros of sequences $\left\{Q_{n}(z)\right\}_{n=0}^{\infty}$ defined by

$$
\sum_{n=0}^{\infty} Q_{n}(z) t^{n}=\frac{1}{1+A(z) t+B(z) t^{2}+C(z) t^{3}}
$$

where $A(z), B(z)$, and $C(z)$ are polynomials in $z$. For a plot of the zeros on the complex plane with specific choices of these polynomials, see Figures 1, 2, 3 , and 4 .

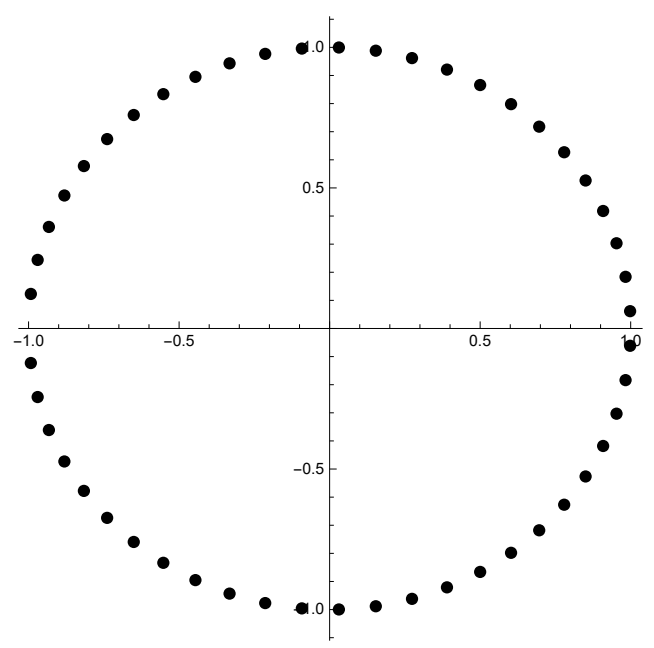

Figure 1. Zeros of $Q_{100}(z): A(z)=1, B(z)=z, C(z)=z$. 


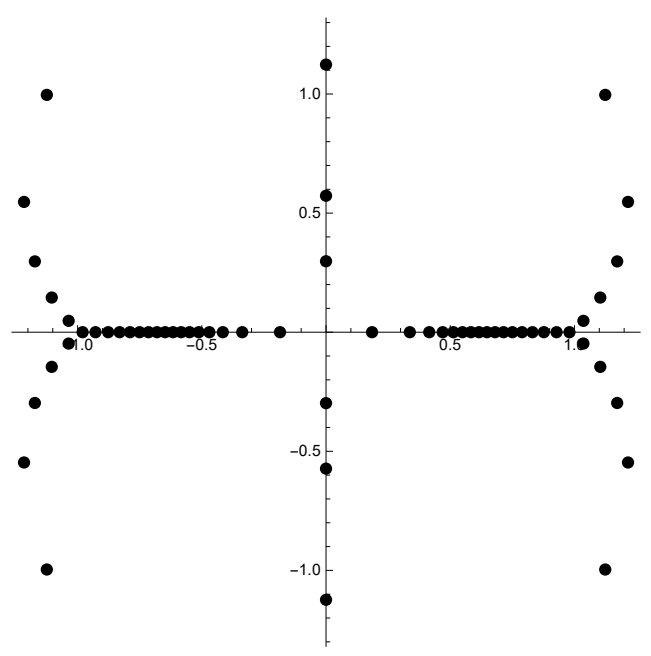

Figure 2. Zeros of $Q_{100}(z): A(z)=1, B(z)=1, C(z)=z^{2}$.

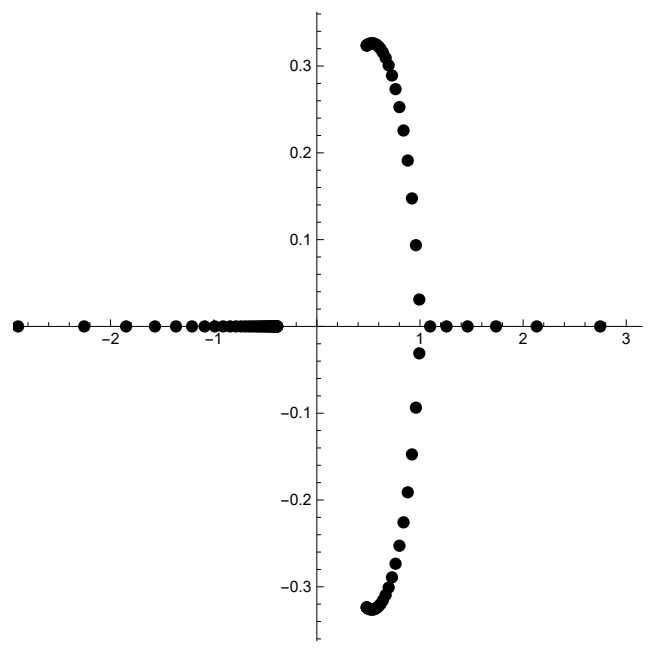

Figure 3. Zeros of $Q_{100}(z): A(z)=1, B(z)=z^{2}, C(z)=z^{3}$. 


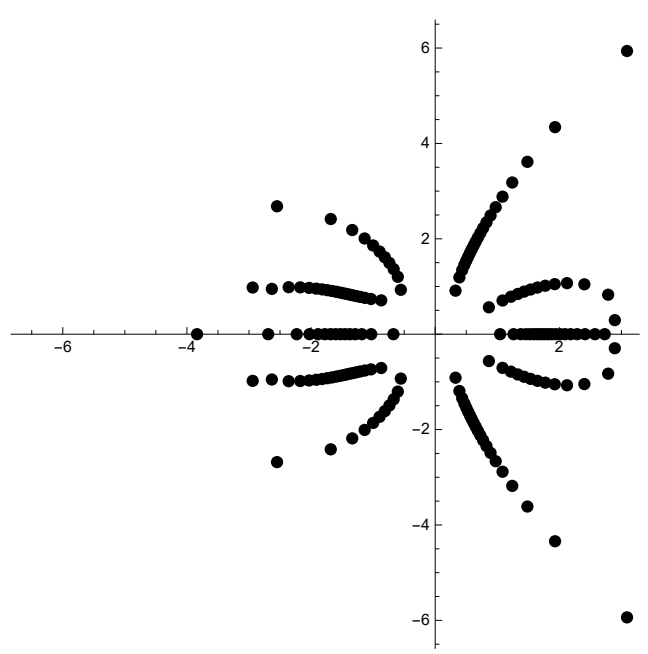

Figure 4. Zeros of $Q_{100}(z): A(z)=1, B(z)=-z^{2}+z+10, C(z)=z^{5}$.

For different choices of $A(z), B(z)$, and $C(z)$, it is clear that some of the zeros of $Q_{n}(z)$ are real, while other zeros are not. We are interested in finding conditions for which $Q_{n}(z)$ is hyperbolic for all $n$. 


\section{THE CAUCHY DIFFERENTIATION FORMULA}

Recall that the method of partial fractions is an elementary way to decompose a product of the form

$$
\frac{1}{\left(t-t_{1}\right)\left(t-t_{2}\right)}
$$

into a sum

$$
\frac{A}{t-t_{1}}+\frac{B}{t-t_{2}}
$$

for some expressions $A$ and $B$, which are constant in $t$. We generalize this procedure in the following lemma.

Lemma 11. Let $t_{1}, t_{2}, \ldots, t_{n} \in \mathbb{C}$ be distinct and $D(t):=\left(t-t_{1}\right)\left(t-t_{2}\right) \ldots\left(t-t_{n}\right)$. Then $\frac{1}{D(t)}$ can be written equivalently as

$$
\sum_{k=1}^{n} \frac{1}{D_{t}\left(t_{k}\right)\left(t-t_{k}\right)} \text {. }
$$

where $D_{t}\left(t_{k}\right)$ is the derivative of $D\left(t_{k}\right)$ with respect to $t$.

Proof. Let

$$
\frac{1}{\left(t-t_{1}\right)\left(t-t_{2}\right) \cdots\left(t-t_{n}\right)}=\frac{A_{1}}{t-t_{1}}+\frac{A_{2}}{t-t_{2}}+\cdots+\frac{A_{n}}{t-t_{n}} .
$$


Then

$$
\begin{aligned}
& 1=A_{1}\left(t-t_{2}\right)\left(t-t_{3}\right) \cdots\left(t-t_{n}\right) \\
& +A_{2}\left(t-t_{1}\right)\left(t-t_{3}\right) \cdots\left(t-t_{n}\right) \\
& \quad \vdots \\
& +A_{n}\left(t-t_{1}\right)\left(t-t_{2}\right) \cdots\left(t-t_{n-1}\right) .
\end{aligned}
$$

First picking $t=t_{1}$, then $t=t_{2}$, and so on until $t=t_{n}$, we obtain

$$
\begin{aligned}
A_{1} & =\frac{1}{\left(t_{1}-t_{2}\right)\left(t_{1}-t_{3}\right) \cdots\left(t_{1}-t_{n}\right)} \\
A_{2} & =\frac{1}{\left(t_{2}-t_{1}\right)\left(t_{2}-t_{3}\right) \cdots\left(t_{2}-t_{n}\right)} \\
& \vdots \\
A_{k} & =\frac{1}{\left(t_{k}-t_{1}\right)\left(t_{k}-t_{2}\right) \cdots\left(t_{k}-t_{k-1}\right)\left(t_{k}-t_{k+1}\right) \cdots\left(t_{k}-t_{n}\right)} \\
& \vdots \\
A_{n} & =\frac{1}{\left(t_{n}-t_{1}\right)\left(t_{n}-t_{2}\right) \cdots\left(t_{n}-t_{n-1}\right)} .
\end{aligned}
$$


Thus $\frac{1}{\left(t-t_{1}\right)\left(t-t_{2}\right) \cdots\left(t-t_{n}\right)}$ is equivalent to

$$
\begin{aligned}
& \frac{1}{\left(t-t_{1}\right)\left(t_{1}-t_{2}\right)\left(t_{1}-t_{3}\right) \cdots\left(t_{1}-t_{n}\right)} \\
+ & \frac{1}{\left(t-t_{2}\right)\left(t_{2}-t_{1}\right)\left(t_{2}-t_{3}\right) \cdots\left(t_{2}-t_{n}\right)} \\
& \vdots \\
+ & \frac{1}{\left(t-t_{k}\right)\left(t_{k}-t_{1}\right)\left(t_{k}-t_{2}\right) \cdots\left(t_{k}-t_{k-1}\right)\left(t_{k}-t_{k+1}\right) \cdots\left(t_{k}-t_{n}\right)} \\
& \vdots \\
+ & \frac{1}{\left(t-t_{n}\right)\left(t_{n}-t_{1}\right)\left(t_{n}-t_{2}\right) \cdots\left(t_{n}-t_{n-1}\right)} \\
= & \sum_{k=1}^{n} \frac{1}{D_{t}\left(t_{k}\right)\left(t-t_{k}\right)} .
\end{aligned}
$$

By the previous lemma, the case where the denominator is quadratic in $t$ with two distinct zeros,

$$
\sum_{n=0}^{\infty} Q_{n}(z) t^{n}=\frac{1}{\left(t-t_{1}\right)\left(t-t_{2}\right)}
$$


can be rewritten in a more useful form as follows.

$$
\begin{aligned}
\sum_{n=0}^{\infty} Q_{n}(z) t^{n} & =\frac{1}{\left(t_{1}-t_{2}\right)\left(t-t_{1}\right)}-\frac{1}{\left(t_{1}-t_{2}\right)\left(t-t_{2}\right)} \\
& =\frac{1}{t_{1}\left(t_{1}-t_{2}\right)\left(\frac{t}{t_{1}}-1\right)}-\frac{1}{t_{2}\left(t_{1}-t_{2}\right)\left(\frac{t}{t_{2}}-1\right)} \\
& =-\frac{1}{t_{1}\left(t_{1}-t_{2}\right)} \sum_{n=0}^{\infty}\left(\frac{t}{t_{1}}\right)^{n}+\frac{1}{t_{2}\left(t_{1}-t_{2}\right)} \sum_{n=0}^{\infty}\left(\frac{t}{t_{2}}\right)^{n} \\
& =\frac{-1}{t_{1}-t_{2}} \sum_{n=0}^{\infty} \frac{t^{n}}{t_{1}^{n+1}}+\frac{1}{t_{1}-t_{2}} \sum_{n=0}^{\infty} \frac{t^{n}}{t_{2}^{n+1}} \\
& =\sum_{n=0}^{\infty}\left[\frac{-1}{t_{1}-t_{2}} \cdot \frac{1}{t_{1}^{n+1}}+\frac{1}{t_{1}-t_{2}} \cdot \frac{1}{t_{2}^{n+1}}\right] t^{n} .
\end{aligned}
$$

Equating coefficients yields

$$
Q_{n}(z)=-\frac{1}{\left(t_{1}-t_{2}\right) t_{1}^{n+1}}-\frac{1}{\left(t_{2}-t_{1}\right) t_{2}^{n+1}}
$$

This method of partial fractions works well for rational functions, but we wish to use a more general approach that can be used for any analytic function in a neighborhood of the origin.

Theorem 12 (Cauchy Differentiation Formula [3]). Let $G$ be an open set and $f: G \rightarrow \mathbb{C}$ an analytic function. If $\gamma$ is a closed rectifiable curve in $G$ such that the winding number $n(\gamma ; w)=0$ for all $w$ in $\mathbb{C} \backslash G$, then for a in $G \backslash \gamma$

$$
f^{(k)}(a) n(\gamma ; a)=\frac{k !}{2 \pi i} \oint_{\gamma} \frac{f(z)}{(z-a)^{k+1}} d z
$$

We use the Cauchy Differentiation Formula to prove an important lemma which will be used throughout this thesis. For $t_{1}$ and $t_{2}$ distinct and 
nonzero, define

$$
\sum_{n=0}^{\infty} Q_{n}(z) t^{n}:=f(t)=\frac{1}{\left(t-t_{1}\right)\left(t-t_{2}\right)} .
$$

If $\gamma$ is the boundary of a disc of radius $R$ containing $0, t_{1}$, and $t_{2}$, we have

$$
\begin{aligned}
\left|\oint_{\gamma} \frac{d t}{\left(t-t_{1}\right)\left(t-t_{2}\right) t^{n+1}}\right| & \leq \oint_{\gamma} \frac{|d t|}{\left|t-t_{1}\right|\left|t-t_{2}\right|\left|t^{n+1}\right|} \\
& \leq \oint_{\gamma} \frac{R}{\left(R-\left|t_{1}\right|\right)\left(R-\left|t_{2}\right|\right) R^{n+1}} d t \\
& =\frac{1}{\left(R-\left|t_{1}\right|\right)\left(R-\left|t_{2}\right|\right) R^{n}} \oint_{\gamma} d t
\end{aligned}
$$

which approaches 0 as $R \rightarrow \infty$. Fix $z \in \mathbb{C}$ and let $\gamma_{1}, \gamma_{2}$, and $\gamma_{3}$ be the boundaries of the disks around $t_{1}, t_{2}$, and 0 respectively. By Theorem 12, we have

$$
\begin{aligned}
\oint_{\gamma} \frac{d t}{\left(t-t_{1}\right)\left(t-t_{2}\right) t^{n+1}} & =\oint_{\gamma_{1}} \frac{\frac{1}{\left(t-t_{2}\right) t^{n+1}}}{t-t_{1}} d t+\oint_{\gamma_{2}} \frac{\frac{1}{\left(t-t_{1}\right) t^{n+1}}}{t-t_{2}} d t+\oint_{\gamma_{3}} \frac{1}{\frac{\left(t-t_{1}\right)\left(t-t_{2}\right)}{t_{2}^{n+1}}} d t \\
& =\frac{2 \pi i}{0 !} \cdot \frac{1}{\left(t_{1}-t_{2}\right) t_{1}^{n+1}}+\frac{2 \pi i}{0 !} \cdot \frac{1}{\left(t_{2}-t_{1}\right) t_{2}^{n+1}}+\frac{2 \pi i}{n !} f^{(n)}(0) \\
& =\frac{2 \pi i}{\left(t_{1}-t_{2}\right) t_{1}^{n+1}}+\frac{2 \pi i}{\left(t_{2}-t_{1}\right) t_{2}^{n+1}}+\frac{2 \pi i}{n !} f^{(n)}(0) .
\end{aligned}
$$

We let $R \rightarrow \infty$ and obtain

$$
0=\frac{2 \pi i}{\left(t_{1}-t_{2}\right) t_{1}^{n+1}}+\frac{2 \pi i}{\left(t_{2}-t_{1}\right) t_{2}^{n+1}}+\frac{2 \pi i}{n !} f^{(n)}(0)
$$

which implies

$$
\frac{f^{(n)}(0)}{n !}=-\frac{1}{\left(t_{1}-t_{2}\right) t_{1}^{n+1}}-\frac{1}{\left(t_{2}-t_{1}\right) t_{2}^{n+1}} .
$$


Since $f(t)$ is analytic at 0 , it has a unique power series in a neighborhood of 0 ,

$$
f(t)=\sum_{n=0}^{\infty} a_{n} t^{n}, \quad \text { where } a_{n}=\frac{f^{(n)}(0)}{n !}
$$

Uniqueness gives $a_{n}=Q_{n}(z) \forall n \geq 0$. So it follows from Equation (8) that

$$
Q_{n}(z)=-\frac{1}{\left(t_{1}-t_{2}\right) t_{1}^{n+1}}-\frac{1}{\left(t_{2}-t_{1}\right) t_{2}^{n+1}}
$$

The expression for a third degree polynomial denominator can be proved in the same way, and we state the result as the following lemma.

Lemma 13. If $t_{1}, t_{2}$, and $t_{3}$ are distinct and nonzero complex numbers, then the equation

$$
\sum_{n=0}^{\infty} Q_{n}(z) t^{n}=\frac{1}{\left(t-t_{1}\right)\left(t-t_{2}\right)\left(t-t_{3}\right)}
$$

implies

$Q_{n}(z)=-\frac{1}{\left(t_{1}-t_{2}\right)\left(t_{1}-t_{3}\right) t_{1}^{n+1}}-\frac{1}{\left(t_{2}-t_{1}\right)\left(t_{2}-t_{3}\right) t_{2}^{n+1}}-\frac{1}{\left(t_{3}-t_{1}\right)\left(t_{3}-t_{2}\right) t_{3}^{n+1}}$. 


\section{APPLICATIONS OF GENERATING FUNCTIONS}

In this section, we show how a generating function can be used to prove the hyperbolicity of polynomials in certain recurrences. We also show how Vieta's formulas are used to derive expressions for the zeros of the denominator of a generating function and the monotone function $z(\theta)$.

Definition 14. The elementary symmetric polynomials in $n$ variables $z_{1}, z_{2}, \ldots, z_{n}$ are defined by

$$
\begin{aligned}
& e_{1}=z_{1}+z_{2}+\cdots+z_{n} \\
& e_{2}=\left(z_{1} z_{2}+z_{1} z_{3}+\cdots+z_{1} z_{n}\right)+\left(z_{2} z_{3}+z_{2} z_{4}+\cdots+z_{2} z_{n}\right)+\cdots+z_{n-1} z_{n} \\
& e_{3}=\left(z_{1} z_{2} z_{3}+z_{1} z_{2} z_{4}+\cdots+z_{1} z_{2} z_{n}\right)+\left(z_{1} z_{3} z_{4}+z_{1} z_{35}+\cdots+z_{1} z_{3} z_{n}\right)+\ldots \\
& \quad+z_{n-2} z_{n-1} z_{n} \\
& \quad \vdots \\
& e_{n}=z_{1} z_{2} \cdots z_{n} .
\end{aligned}
$$

Theorem 15 (Vieta's Formulas). Let $P(z)=a_{n} z^{n}+a_{n-1} z^{n-1}+\cdots+a_{1} z+a_{0}$ be a polynomial with $n$ zeros $z_{1}, z_{2}, \ldots, z_{n}$. Then

$$
\begin{aligned}
e_{1} & =-\frac{a_{n-1}}{a_{n}} \\
e_{2} & =\frac{a_{n-2}}{a_{n}} \\
\vdots & \\
e_{k} & =(-1)^{k} \frac{a_{n-k}}{a_{n}}
\end{aligned}
$$


Proof. Let $z_{1}, z_{2}, \ldots, z_{n}$ be the zeros of $P(z)$. Then

$$
\begin{aligned}
P(z)= & a_{n}\left(z-z_{1}\right)\left(z-z_{2}\right)\left(z-z_{3}\right)\left(z-z_{4}\right) \cdots\left(z-z_{n}\right) \\
= & a_{n}\left[z^{n}-\left(z_{n}+z_{n-1}+z_{n-2}+\cdots+z_{1}\right) z^{n-1}\right. \\
& +\left(z_{n} z_{n-1}+z_{n} z_{n-2}+\cdots+z_{2} z_{1}\right) z^{n-2} \\
& -\left(z_{n} z_{n-1} z_{n-2}+z_{n} z_{n-1} z_{n-3}+\cdots+z_{3} z_{2} z_{1}\right) z^{n-3} \\
& +\left(z_{n} z_{n-1} z_{n-2} z_{n-3}+z_{n} z_{n-1} z_{n-2} z_{n-4}+\cdots+z_{4} z_{3} z_{2} z_{1}\right) z^{n-4} \\
& \vdots \\
& +\left(z_{n} z_{n-1} \cdots z_{2}+z_{n} z_{n-1} \cdots z_{3}+\cdots+z_{2} z_{3} \cdots z_{n}\right) z \\
& \left.+\left(z_{1} z_{2} z_{3} z_{4} \cdots z_{n}\right)\right] \\
= & a_{n} z^{n}-a_{n} e_{1} z^{n-1}+a_{n} e_{2} z^{n-2}-a_{n} e_{3} z^{n-3}+\cdots+a_{n} e_{n-1} z+a_{n} e_{n} .
\end{aligned}
$$

Matching coefficients gives

$$
\begin{aligned}
e_{1} & =-\frac{a_{n-1}}{a_{n}} \\
e_{2} & =\frac{a_{n-2}}{a_{n}} \\
\vdots & \\
e_{k} & =(-1)^{k} \frac{a_{n-k}}{a_{n}}
\end{aligned}
$$

which concludes the proof. 
Recall from Lemma 10 that the sequence of Chebyshev polynomials in Definition 8 can be equivalently defined by the generating relation

$$
\sum_{n=0}^{\infty} U_{n}(z) t^{n}=\frac{1}{1-2 z t+t^{2}}
$$

If we let $t_{U_{1}}=e^{-i \theta}$ and $t_{U_{2}}=e^{i \theta}$, then $t_{U_{1}}+t_{U_{2}}=2 \cos \theta$ and $t_{U_{1}} t_{U_{2}}=1$. If we further let $z_{U}=\cos \theta$, then $t_{U_{1}}$ and $t_{U_{2}}$ satisfy Vieta's formulas for the polynomial $1-2 z_{U} t+t^{2}$ and hence they are the zeros of $1-2 z_{U} t+t^{2}$. Since $t_{U_{1}} \neq t_{U_{2}} \neq 0$, we have by Lemma 13 that

$$
U_{n}(z)=\frac{-1}{\left(t_{U_{1}}-t_{U_{2}}\right) t_{U_{1}}^{n+1}}+\frac{1}{\left(t_{U_{1}}-t_{U_{2}}\right) t_{U_{2}}^{n+1}} .
$$

We use Equation (9) to derive the function $g_{U_{n}}$ as follows.

$$
\begin{aligned}
U_{n}(z) & =\frac{1}{t_{U_{2}}-t_{U_{1}}}\left(\frac{1}{t_{U_{1}}^{n+1}}-\frac{1}{t_{U_{2}}^{n+1}}\right) \\
& =\frac{1}{t_{U_{2}}-t_{U_{1}}}\left(\frac{t_{U_{2}}^{n+1}-t_{U_{1}}^{n+1}}{t_{U_{1}}^{n+1} t_{U_{2}}^{n+1}}\right) \\
& =\frac{1}{e^{i \theta}-e^{-i \theta}}\left(\frac{e^{i(n+1) \theta}-e^{-i(n+1) \theta}}{e^{-i(n+1) \theta} e^{i(n+1) \theta}}\right) \\
& =\frac{e^{i(n+1) \theta}-e^{-i(n+1) \theta}}{e^{i \theta}-e^{-i \theta}} \\
& =\frac{\sin ((n+1) \theta)}{\sin \theta} \\
& =g_{U_{n}} .
\end{aligned}
$$

Hence we obtain Equations (1) and (2), which were used to prove Proposition 8. 
The generating function is very useful for analyzing the zeros of recursive sequences of polynomials. As an example, consider the sequence $\left\{H_{n}(z)\right\}_{n=0}^{\infty}$ generated by

$$
\sum_{n=0}^{\infty} H_{n}(z) t^{n}=\frac{1}{1+t+z t^{2}}
$$

See Figure 5 for a plot of the zeros of $H_{100}(z)$ on the complex plane.

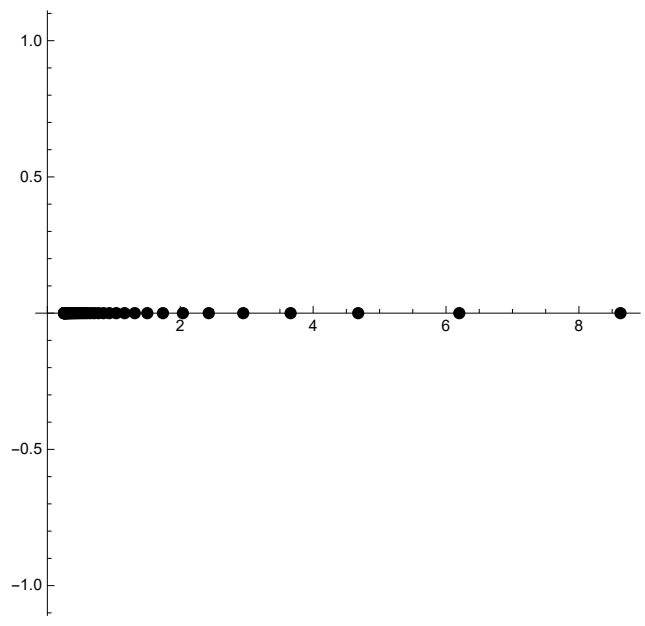

Figure 5. Zeros of $H_{100}(z): A(z)=1, B(z)=z, C(z)=0$.

Proposition 16. All the zeros of the sequence $\left\{H_{n}(z)\right\}_{n=0}^{\infty}$ generated by

$$
\sum_{n=0}^{\infty} H_{n}(z) t^{n}=\frac{1}{1+t+z t^{2}}
$$

are real and lie on the interval $(1 / 4, \infty)$, where they are dense as $n \rightarrow \infty$. 
Proof. For $\theta \in(0, \pi / 2)$, define the functions

$$
\begin{aligned}
z_{H}(\theta) & :=\frac{1}{4} \sec ^{2} \theta, \\
\tau_{H}(\theta) & :=-2 \cos \theta, \\
t_{H_{1}}(\theta) & :=\tau_{H}(\theta) e^{-i \theta}, \\
t_{H_{2}}(\theta) & :=\tau_{H}(\theta) e^{i \theta} .
\end{aligned}
$$

Note that $z_{H}$ is monotone on $(0, \pi / 2)$. We will show that for $\theta \in(0, \pi / 2), t_{H_{1}}$ and $t_{H_{2}}$ are the zeros of

$$
1+t+z_{H} t^{2}
$$

Observe that

$$
\begin{aligned}
t_{H_{1}}+t_{H_{2}} & =\tau_{H} e^{-i \theta}+\tau_{H} e^{i \theta}=\tau_{H}\left(e^{-i \theta}+e^{i \theta}\right)=\tau_{H}(2 \cos \theta) \\
& =-2 \cos \theta(2 \cos \theta)=-4 \cos ^{2} \theta=-\frac{1}{z_{H}} .
\end{aligned}
$$

Furthermore,

$$
\begin{aligned}
t_{H_{1}} t_{H_{2}} & =\tau_{H} e^{-i \theta} \tau_{H} e^{i \theta}=\tau_{H}^{2} e^{0}=(2 \cos \theta)^{2} \\
& =4 \cos ^{2} \theta=\frac{1}{z_{H}} .
\end{aligned}
$$

Since $t_{H_{1}}$ and $t_{H_{2}}$ satisfy

$$
t_{H_{1}}+t_{H_{2}}=-\frac{1}{z_{H}} \text { and } t_{H_{1}} t_{H_{2}}=\frac{1}{z_{H}},
$$


it must be true that they are the zeros of $1+t+z_{H} t^{2}$ by Vieta's formulas. By definition, $t_{H_{1}}$ and $t_{H_{2}}$ are distinct and nonzero, so we can apply Lemma 13 to obtain

$$
H_{n}(z)=\frac{1}{t_{H_{2}}-t_{H_{1}}}\left(\frac{1}{t_{H_{1}}^{n+1}}-\frac{1}{t_{H_{2}}^{n+1}}\right) .
$$

When we substitute $t_{H_{1}}=\tau_{H} e^{-i \theta}$ and $t_{H_{2}}=\tau_{H} e^{i \theta}$ into (10), we obtain

$$
\begin{aligned}
H_{n}\left(z_{H}\right) & =\frac{1}{\tau_{H} e^{i \theta}-\tau_{H} e^{-i \theta}}\left(\frac{\left(\tau_{H} e^{i \theta}\right)^{n+1}-\left(\tau_{H} e^{-i \theta}\right)^{n+1}}{\left(\tau_{H} e^{-i \theta}\right)^{n+1}\left(\tau_{H} e^{i \theta}\right)^{n+1}}\right) \\
& =\frac{1}{\tau_{H}} \cdot \frac{1}{e^{i \theta}-e^{-i \theta}}\left(\frac{\tau_{H}^{n+1}\left(e^{i(n+1) \theta}-e^{-i(n+1) \theta}\right)}{\tau_{H}^{2 n+2}}\right) \\
& =\frac{1}{\tau_{H}^{n+2}} \cdot \frac{e^{i(n+1) \theta}-e^{-i(n+1) \theta}}{e^{i \theta}-e^{-i \theta}} \\
& =\frac{1}{\tau_{H}^{n+2}} \cdot \frac{\sin ((n+1) \theta)}{\sin (\theta)} \\
& =\frac{1}{\tau_{H}^{n} \tau_{H}^{2}} \cdot \frac{\sin ((n+1) \theta)}{\sin (\theta)} \\
& =\frac{1}{\tau_{H}^{n}} \cdot z \cdot \frac{\sin ((n+1) \theta)}{\sin (\theta)} \\
& =\frac{1}{\tau_{H}^{n}} \cdot \frac{1}{4} \sec { }^{2} \theta \cdot \frac{\sin ((n+1) \theta)}{\sin (\theta)} \\
& =\frac{1}{\tau_{H}^{n}} \cdot \frac{\sin ((n+1) \theta)}{2 \sin (2 \theta) \cos \theta} .
\end{aligned}
$$

Note that $\frac{1}{\tau_{H}^{n}} \in \mathbb{R}$ so this number does not affect the zeros of the expression, so define

$$
g_{H_{n}}(\theta):=\frac{\sin ((n+1) \theta)}{\sin (2 \theta) \cos \theta}
$$


For $\theta \in(0, \pi / 2)$, we find the zeros of $H_{n}(z)$ by finding the zeros of Equation (11) to be

$$
\theta=\frac{k \pi}{n+1}, k \in\{1,2,3, \ldots,\lfloor n / 2\rfloor\} .
$$

Hence there are $\left\lfloor\frac{n}{2}\right\rfloor$ distinct solutions of $g_{H_{n}}=0$ on $(0, \pi / 2)$. Note that each distinct solution of $g_{H_{n}}=0$ on $(0, \pi / 2)$ gives a distinct zero of $H_{n}\left(z_{H}(\theta)\right)$ via the equation

$$
z_{H}(\theta)=\frac{1}{4} \sec ^{2} \theta
$$

since $z_{H}$ is monotone on $(0, \pi / 2)$. Furthermore, $z_{H} \in(1 / 4, \infty)$ for $\theta \in(0, \pi / 2)$. We will now show that the degree of $H_{n}(z)$ is at most $\left\lfloor\frac{n}{2}\right\rfloor$ by forming the recurrence relation for $H_{n}(z)$. By Lemma 10, we get that the sequence $\left\{H_{n}(z)\right\}_{n=0}^{\infty}$ satisfies the recurrence relation

$$
H_{n}(z)+H_{n-1}(z)+z H_{n-2}(z)=0
$$

where $H_{0}(z)=1$ and $H_{-n}(z)=0$. We proceed by strong induction on $n$ to show that the degree of $H_{n}(z)$ is at most $\left\lfloor\frac{n}{2}\right\rfloor$.

In the base case where $n=0$, we have $H_{0}(z)=1$. So the degree of $H_{0}(z)$ is $0 \leq\left\lfloor\frac{0}{2}\right\rfloor$.

Suppose now that

$$
\operatorname{deg}\left(H_{n-1}(z)\right) \leq\left\lfloor\frac{n-1}{2}\right\rfloor \text { and } \operatorname{deg}\left(H_{n-2}(z)\right) \leq\left\lfloor\frac{n-2}{2}\right\rfloor
$$

Then

$$
\operatorname{deg}\left(H_{n}(z)\right) \leq \max \left\{\left\lfloor\frac{n-1}{2}\right\rfloor,\left\lfloor\frac{n-2}{2}\right\rfloor+1\right\} .
$$


If $n$ is odd, then $n=2 a+1$ for some $a \in \mathbb{N}$. So

$$
\left\lfloor\frac{n-1}{2}\right\rfloor=\left\lfloor\frac{2 a+1-1}{2}\right\rfloor=a=\left\lfloor\frac{2 a+1}{2}\right\rfloor=\left\lfloor\frac{n}{2}\right\rfloor .
$$

Furthermore,

$$
\left\lfloor\frac{n-2}{2}\right\rfloor+1=\left\lfloor\frac{2 a-1}{2}\right\rfloor+1=a=\left\lfloor\frac{2 a+1}{2}\right\rfloor=\left\lfloor\frac{n}{2}\right\rfloor .
$$

If $n$ is even, then $n=2 a$ for some $a \in \mathbb{N}$. So

$$
\left\lfloor\frac{n-1}{2}\right\rfloor=\left\lfloor\frac{2 a-1}{2}\right\rfloor=a-1<a=\left\lfloor\frac{2 a}{2}\right\rfloor=\left\lfloor\frac{n}{2}\right\rfloor .
$$

Furthermore,

$$
\left\lfloor\frac{n-2}{2}\right\rfloor+1=\left\lfloor\frac{2 a-2}{2}\right\rfloor+1=a=\left\lfloor\frac{2 a}{2}\right\rfloor=\left\lfloor\frac{n}{2}\right\rfloor .
$$

Hence by induction, we see that $\operatorname{deg}\left(H_{n}(z)\right)$ is at most $\left\lfloor\frac{n}{2}\right\rfloor$.

Since we found that there are exactly $\left\lfloor\frac{n}{2}\right\rfloor$ zeros of $H_{n}(z)$ on $(1 / 4, \infty)$, we have by the Fundamental Theorem of Algebra that these are the only zeros of $H_{n}(z)$. Thus all of the zeros of $H_{n}(z)$ lie on the real interval $(1 / 4, \infty)$, and since $z_{H}:(0, \pi / 2) \rightarrow(1 / 4, \infty)$ is a continuous bijective map, we have by Theorem 4 that the set of zeros of $H_{n}(z)$ is dense on $(1 / 4, \infty)$ as $n \rightarrow \infty$. 
The sequence $\left\{H_{n}(z)\right\}_{n=0}^{\infty}$ defined in Proposition 10 is actually related to a more general sequence. Consider the sequence $\left\{Q_{n}(z)\right\}_{n=0}^{\infty}$ defined by

$$
\sum_{n=0}^{\infty} Q_{n}(z) t^{n}=\frac{1}{1+A(z) t+B(z) t^{2}}
$$

If we make the substitution $t \rightarrow \frac{t}{A(z)}$, then Equation (12) becomes

$$
\sum_{n=0}^{\infty} \frac{Q_{n}(z)}{A^{n}(z)} t^{n}=\frac{1}{1+t+\frac{B(z)}{A^{2}(z)} t^{2}} .
$$

If we next make the substitution $\frac{B(z)}{A^{2}(z)} \rightarrow z$, then the right hand side of Equation (13) becomes the generating function for $H_{n}(z)$, so we have

$$
Q_{n}(z)=A^{n}(z) H_{n}\left(\frac{B(z)}{A^{2}(z)}\right)
$$

So the zeros of $Q_{n}(z)$ which are not zeros of $A(z)$ must be zeros of $H_{n}\left(\frac{B(z)}{A^{2}(z)}\right)$. By Proposition $10, z_{0}$ is a zero of $H_{n}(z)$ if and only if $\frac{B\left(z_{0}\right)}{A^{2}\left(z_{0}\right)}>\frac{1}{4}$. This procedure was proved by Tran in [9] as the following theorem.

Theorem 17. Let $\left\{Q_{n}(z)\right\}_{n=0}^{\infty}$ be a sequence of polynomials whose generating function is

$$
\sum_{n=0}^{\infty} Q_{n}(z) t^{n}=\frac{1}{1+A(z) t+B(z) t^{2}}
$$


where $A(z)$ and $B(z)$ are polynomials in $z$ with complex coefficients. The roots of $Q_{n}(z)$ which satisfy $B(z) \neq 0$ lie on the curve $\mathcal{C}_{2}$ defined by

$$
\operatorname{Im} \frac{A^{2}(z)}{B(z)}=0 \quad \text { and } \quad 0 \leq \operatorname{Re} \frac{A^{2}(z)}{B(z)} \leq 4
$$

and are dense there as $n \rightarrow \infty$.

As an example, let us apply Theorem 17 to the sequence of Chebyshev polynomials $\left\{U_{n}(z)\right\}_{n=0}^{\infty}$. Recall that the sequence $\left\{U_{n}(z)\right\}_{n=0}^{\infty}$ can be defined by the generating relation

$$
\sum_{n=0}^{\infty} U_{n}(z) t^{n}=\frac{1}{1-2 z t+t^{2}}
$$

where $A(z)=-2 z$ and $B(z)=1$. By Theorem 17 , the nonzero zeros of $U_{n}(z)$ lie on the curve defined by

$$
\operatorname{Im} 4 z^{2}=0 \quad \text { and } \quad 0 \leq \operatorname{Re} 4 z^{2} \leq 4
$$

which implies that the zeros are real and lie on the interval $[-1,1]$. 


\section{THE CUBIC DENOMINATOR}

The main goal of this thesis is to study the zeros of the sequence $\left\{Q_{n}(z)\right\}_{n=0}^{\infty}$ generated by

$$
\sum_{n=0}^{\infty} Q_{n}(z) t^{n}=\frac{1}{1+A(z) t+B(z) t^{2}+C(z) t^{3}}
$$

where $A(z), B(z)$ and $C(z)$ are nonzero linear complex-valued polynomials in $z$ with real coefficients. As a simple example of the cubic denominator, consider the choices $A(z)=1, B(z)=0$, and $C(z)=z$. With these choices we obtain the sequence $\left\{K_{n}(z)\right\}_{n=0}^{\infty}$ generated by

$$
\sum_{n=0}^{\infty} K_{n}(z) t^{n}=\frac{1}{1+t+z t^{3}}
$$

See Figure 6 for a plot of the zeros of $K_{100}(z)$ on the complex plane.

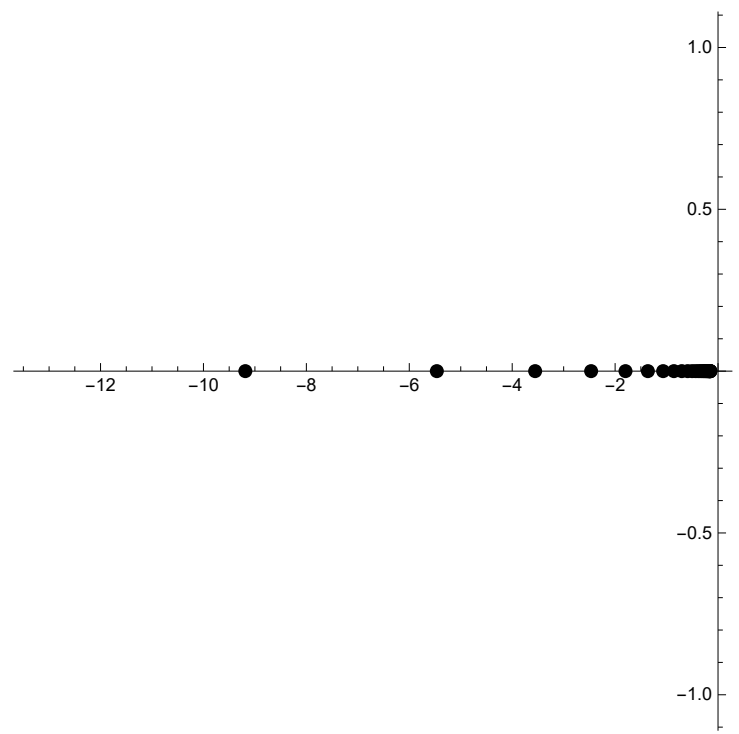

Figure 6. Zeros of $K_{100}(z): A(z)=1, B(z)=0, C(z)=z$. 
All the zeros appear to lie on the real axis. Before we prove that they do in fact lie on the real line, we first prove an important lemma that will help us define our sequence $\left\{g_{n}(\theta)\right\}_{n=0}^{\infty}$ for the next two recurrences defined in this thesis.

Lemma 18. Let $Q_{n}(z)$ be defined as in Equation (14). Suppose $z \in \mathbb{R} \backslash\{0\}$ such that the zeros of $1+A(z) t+B(z) t^{2}+C(z) t^{3}$ are $t_{1}=\tau e^{-i \theta}, t_{2}=\tau e^{i \theta}$, and $t_{3}=\tau \zeta$, where $\tau, \zeta \in \mathbb{R} \backslash\{0\}$. Then $z$ is a zero of $Q_{n}(z)$ if and only if

$$
\frac{(\zeta-\cos \theta) \sin (n+1) \theta}{\sin \theta}-\cos (n+1) \theta+\frac{1}{\zeta^{n+1}}=0 .
$$

Proof. By Lemma 13, $z$ is a zero of $Q_{n}(z)$ if and only if

$$
\frac{1}{\left(t_{1}-t_{2}\right)\left(t_{1}-t_{3}\right) t_{1}^{n+1}}+\frac{1}{\left(t_{2}-t_{1}\right)\left(t_{2}-t_{3}\right) t_{2}^{n+1}}+\frac{1}{\left(t_{3}-t_{1}\right)\left(t_{3}-t_{2}\right) t_{3}^{n+1}}=0
$$

Replacing $t_{1}, t_{2}$, and $t_{3}$ with $\tau e^{-i \theta}, \tau e^{i \theta}$, and $\tau \zeta$, respectively, and multiplying by $\tau^{n+3}$ gives

$$
\begin{aligned}
0 & =\frac{1}{\left(e^{-i \theta}-e^{i \theta}\right)\left(e^{-i \theta}-\zeta\right)\left(e^{-i \theta}\right)^{n+1}} \\
& -\frac{1}{\left(e^{-i \theta}-e^{i \theta}\right)\left(e^{i \theta}-\zeta\right)\left(e^{i \theta}\right)^{n+1}} \\
& +\frac{1}{\left(\zeta-e^{-i \theta}\right)\left(\zeta-e^{i \theta}\right)(\zeta)^{n+1}},
\end{aligned}
$$

which is equivalent to

$$
0=\frac{1}{e^{-i \theta}-e^{i \theta}}\left(\frac{1}{\left(e^{-i \theta}-\zeta\right) e^{-i(n+1) \theta}}-\frac{1}{\left(e^{i \theta}-\zeta\right) e^{i(n+1) \theta}}\right)+\frac{1}{\left|\zeta-e^{-i \theta}\right|^{2} \zeta^{n+1}} .
$$


If we let $E=\left(e^{-i \theta}-\zeta\right) e^{-i(n+1) \theta}$, then $\bar{E}=\left(e^{i \theta}-\zeta\right) e^{i(n+1) \theta}$, and the right hand side of Equation (15) becomes

$$
\begin{aligned}
& \frac{1}{e^{-i \theta}-e^{i \theta}}\left(\frac{1}{E}-\frac{1}{\bar{E}}\right)+\frac{1}{\left|\zeta-e^{-i \theta}\right|^{2} \zeta^{n+1}} \\
= & -\frac{1}{2 i \sin \theta}\left(\frac{\bar{E}-E}{|E|^{2}}\right)+\frac{1}{|E|^{2} \zeta^{n+1}} .
\end{aligned}
$$

Since Equation (15) is equal to zero, we can multiply both sides by $|E|^{2}$ without affecting the zeros and the right hand side becomes

$$
\begin{aligned}
& -\frac{1}{2 i \sin \theta}(2 i \operatorname{Im}(A))+\frac{1}{\zeta^{n+1}} \\
= & -\frac{1}{\sin \theta}(\cos \theta \sin (n+1) \theta+\sin \theta \cos (n+1) \theta-\zeta \sin (n+1) \theta)+\frac{1}{\zeta^{n+1}} \\
= & -\frac{(\cos \theta-\zeta) \sin (n+1) \theta+\cos (n+1) \theta \sin \theta}{\sin \theta}+\frac{1}{\zeta^{n+1}} \\
= & \frac{(\zeta-\cos \theta) \sin (n+1) \theta}{\sin \theta}-\cos (n+1) \theta+\frac{1}{\zeta^{n+1}} .
\end{aligned}
$$

Hence $z$ is a zero of $Q_{n}(z)$ if and only if

$$
\frac{(\zeta-\cos \theta) \sin (n+1) \theta}{\sin \theta}-\cos (n+1) \theta+\frac{1}{\zeta^{n+1}}=0 .
$$

With Lemma 18 at our disposal, we are ready to prove the hyperbolicity of $\left\{K_{n}(z)\right\}_{n=0}^{\infty}$.

Proposition 19. All the zeros of the sequence $\left\{K_{n}(z)\right\}_{n=0}^{\infty}$ generated by

$$
\sum_{n=0}^{\infty} K_{n}(z) t^{n}=\frac{1}{1+t+z t^{3}}
$$


are real and lie on the interval $(-\infty,-4 / 27)$, where they are dense as $n \rightarrow \infty$.

Proof. By Lemma 10, we get that the sequence $\left\{K_{n}(z)\right\}_{n=0}^{\infty}$ satisfies the recurrence relation

$$
K_{n}(z)=-K_{n-1}(z)-z K_{n-3}(z),
$$

where $K_{0}(z)=1$ and $K_{-n}(z)=0$. We first show that the degree of $K_{n}(z)$ is at most $\left\lfloor\frac{n}{3}\right\rfloor$ by induction on $n$.

In the base case where $n=0, K_{0}(z)=1$. So the degree of $K_{0}(z)$ is $0 \leq\left\lfloor\frac{0}{3}\right\rfloor$.

Suppose

$$
\operatorname{deg}\left(K_{n-1}(z)\right)=\left\lfloor\frac{n-1}{3}\right\rfloor \text { and } \operatorname{deg}\left(K_{n-3}(z)\right)=\left\lfloor\frac{n-3}{3}\right\rfloor
$$

Then

$$
\operatorname{deg}\left(K_{n}(z)\right) \leq \max \left\{\left\lfloor\frac{n-1}{3}\right\rfloor,\left\lfloor\frac{n-3}{3}\right\rfloor+1\right\}
$$

If $n$ is a multiple of 3 , then $n=3 a$ for some $a \in \mathbb{N}$. So

$$
\left\lfloor\frac{n-1}{3}\right\rfloor=\left\lfloor\frac{3 a-1}{3}\right\rfloor=a-1<a=\left\lfloor\frac{3 a}{3}\right\rfloor=\left\lfloor\frac{n}{3}\right\rfloor .
$$

Futhermore,

$$
\left\lfloor\frac{n-3}{3}\right\rfloor+1=\left\lfloor\frac{3 a-3}{3}\right\rfloor+1=a=\left\lfloor\frac{3 a}{a}\right\rfloor=\left\lfloor\frac{n}{3}\right\rfloor .
$$


If $n$ is not a multiple of 3 , then $n=3 a+1$ or $n=3 a+2$ for some $a \in \mathbb{N}$. If $n=3 a+1$, we have

$$
\left\lfloor\frac{n-1}{3}\right\rfloor=\left\lfloor\frac{3 a+1-1}{3}\right\rfloor=a=\left\lfloor\frac{3 a+1}{3}\right\rfloor=\left\lfloor\frac{n}{3}\right\rfloor .
$$

Furthermore,

$$
\left\lfloor\frac{n-3}{3}\right\rfloor+1=\left\lfloor\frac{3 a-2}{3}\right\rfloor+1=a=\left\lfloor\frac{3 a+1}{3}\right\rfloor=\left\lfloor\frac{n}{3}\right\rfloor .
$$

If $n=3 a+2$, we have

$$
\left\lfloor\frac{n-1}{3}\right\rfloor=\left\lfloor\frac{3 a+2-1}{3}\right\rfloor=a=\left\lfloor\frac{3 a+2}{3}\right\rfloor=\left\lfloor\frac{n}{3}\right\rfloor .
$$

Furthermore,

$$
\left\lfloor\frac{n-3}{3}\right\rfloor+1=\left\lfloor\frac{3 a-1}{3}\right\rfloor+1=a=\left\lfloor\frac{3 a+2}{3}\right\rfloor=\left\lfloor\frac{n}{3}\right\rfloor .
$$

Hence, by induction, we see that the degree of $K_{n}(z)$ is at most $\left\lfloor\frac{n}{3}\right\rfloor$. 
For $\theta \in(0, \pi / 3)$, define the functions

$$
\begin{aligned}
z_{K}(\theta) & :=\frac{4 \cos ^{2} \theta}{\left(1-4 \cos ^{2} \theta\right)^{3}}, \\
\zeta_{K}(\theta) & :=-2 \cos \theta \\
\tau_{K}(\theta) & :=\sqrt[3]{\frac{1}{2 z_{K}(\theta) \cos \theta}}, \\
t_{K_{1}}(\theta) & :=\tau_{K}(\theta) e^{-i \theta} \\
t_{K_{2}}(\theta) & :=\tau_{K}(\theta) e^{i \theta}, \\
t_{K_{3}}(\theta) & :=\tau_{K}(\theta) \zeta_{K}(\theta) .
\end{aligned}
$$

Since $\cos \theta \neq 0$ and $z_{K} \neq 0$ on $(0, \pi / 3)$, we have that $\tau_{K}$ is well-defined. We differentiate $z_{K}$ to show that it is monotone on $(0, \pi / 3)$ :

$$
z_{K}^{\prime}(\theta)=\frac{-4 \sin (2 \theta)\left(1+8 \cos ^{2} \theta\right)}{\left(1-4 \cos ^{2} \theta\right)^{4}} .
$$

Since $1+8 \cos ^{2} \theta>0,\left(1-4 \cos ^{2} \theta\right)^{4}>0$, and $-4 \sin (2 \theta)<0$ on $(0, \pi / 3)$, we have that $z_{K}^{\prime}(\theta)<0$ on $(0, \pi / 3)$, and hence $z_{K}$ is decreasing on $(0, \pi / 3)$.

We now show that $t_{K_{1}}, t_{K_{2}}$, and $t_{K_{3}}$ satisfy Vieta's formulas. Observe

$$
\begin{aligned}
t_{K_{1}}+t_{K_{2}}+t_{K_{3}} & =\tau_{K} e^{-i \theta}+\tau_{K} e^{i \theta}+\tau_{K} \zeta_{K} \\
& =\tau_{K}\left(2 \cos \theta+\zeta_{K}\right) \\
& =\tau_{K}(2 \cos \theta-2 \cos \theta) \\
& =0 .
\end{aligned}
$$


Also,

$$
\begin{aligned}
t_{K_{1}} t_{K_{2}}+t_{K_{1}} t_{K_{3}}+t_{K_{2}} t_{K_{3}} & =\tau_{K} e^{-i \theta} \tau_{K} e^{i \theta}+\tau_{K} e^{-i \theta} \tau_{K} \zeta_{K}+\tau_{K} e^{i \theta} \tau_{K} \zeta_{K} \\
& =\tau_{K}^{2}\left(1+\zeta_{K} e^{-i \theta}+\zeta_{K} e^{i \theta}\right. \\
& =\tau_{K}^{2}\left(1+2 \zeta_{K} \cos \theta\right) \\
& =\tau_{K}^{2}\left(1-4 \cos ^{2} \theta\right) \\
& =\left(\frac{1}{2 z_{K} \cos \theta}\right)^{\frac{2}{3}}\left(1-4 \cos ^{2} \theta\right) \\
& =\frac{1-4 \cos { }^{2} \theta}{\left(2 z_{K} \cos ^{2}\right)^{\frac{2}{3}}} \\
& =\frac{1-4 \cos ^{2} \theta}{\left(\frac{8 \cos ^{3} \theta}{\left(1-4 \cos ^{2} \theta\right)^{3}}\right)^{\frac{2}{3}}} \\
& =\frac{\left(1-4 \cos ^{2} \theta\right)^{3}}{4 \cos ^{2} \theta} \\
& =\frac{1}{z_{K}}
\end{aligned}
$$

Finally,

$$
\begin{aligned}
t_{K_{1}} t_{K_{2}} t_{K_{3}} & =\tau_{K} e^{-i \theta} \tau_{K} e^{i \theta} \tau_{K} \zeta_{K} \\
& =\tau_{K}^{3} \zeta_{K} \\
& =\left(\frac{1}{2 z_{K} \cos \theta}\right)(-2 \cos \theta) \\
& =-\frac{1}{z_{K}}
\end{aligned}
$$

Since $t_{K_{1}}, t_{K_{2}}$, and $t_{K_{3}}$ are distinct and nonzero on $(0, \pi / 3)$, and they satisfy Vieta's formulas for the polynomial $1+t+z_{K} t^{3}$, we get that they are the zeros of $1+t+z_{K} t^{3}$. 
By Lemma 18, $z$ is a zero of $K_{n}(z)$ if and only if

$$
\frac{(\zeta-\cos \theta) \sin (n+1) \theta}{\sin \theta}-\cos (n+1) \theta+\frac{1}{\zeta^{n+1}}=0,
$$

or equivalently,

$$
-\frac{3 \cos \theta \sin (n+1) \theta}{\sin \theta}-\cos (n+1) \theta+\frac{1}{(-2 \cos \theta)^{n+1}}=0
$$

Define

$$
g_{K_{n}}(\theta):=-\frac{3 \cos \theta \sin (n+1) \theta}{\sin \theta}-\cos (n+1) \theta+\frac{1}{(-2 \cos \theta)^{n+1}} .
$$

Note that when $\cos (n+1) \theta= \pm 1, \sin (n+1) \theta=0$, and since $|-2 \cos \theta|>1$ on $(0, \pi / 3)$, the sign of $g_{K_{n}}$ can be determined by $\cos (n+1) \theta$. Since $|-2 \cos \theta|>1$, we know $\left|\frac{1}{(-2 \cos \theta)^{n+1}}\right|<1$. So when $\cos (n+1) \theta=1$, $g_{K_{n}}<0$, and when $\cos (n+1) \theta=-1, g_{K_{n}}>0$. For $\theta \in(0, \pi / 3)$,

$$
\begin{aligned}
\cos (n+1) \theta= \pm 1 & \Longrightarrow \theta=\frac{k \pi}{n+1}, k \in \mathbb{Z} \\
& \Longrightarrow k \in\{1,2, \ldots,\lfloor n / 3\rfloor\}
\end{aligned}
$$

This means that $g_{K_{n}}$ changes sign $\lfloor n / 3\rfloor-1$ times, so we have found $\lfloor n / 3\rfloor-1$ zeros of $g_{K_{n}}$. Since $\operatorname{deg}\left(K_{n}(z)\right)$ is at most $\lfloor n / 3\rfloor$, the Fundamental Theorem of Algebra implies that there could be one more zero. We claim that 
the one missing zero lies on the interval $\left(0, \frac{\pi}{n+1}\right)$. Observe

$$
\begin{aligned}
\lim _{\theta \rightarrow 0^{+}} g_{K_{n}} & =\lim _{\theta \rightarrow 0^{+}}\left(-\frac{3 \cos \theta \sin (n+1) \theta}{\sin \theta}-\cos (n+1) \theta+\frac{1}{(-2 \cos \theta)^{n+1}}\right) \\
& =-3(n+1)-1+\frac{1}{(-2)^{n+1}} \\
& <0 .
\end{aligned}
$$

Also,

$$
\begin{aligned}
\lim _{\theta \rightarrow\left(\frac{\pi}{n+1}\right)^{-}} g_{K_{n}} & =\lim _{\theta \rightarrow\left(\frac{\pi}{n+1}\right)}\left(-\frac{3 \cos \theta \sin (n+1) \theta}{\sin \theta}-\cos (n+1) \theta+\frac{1}{(-2 \cos \theta)^{n+1}}\right) \\
& =-\frac{3 \cos \left(\frac{\pi}{n+1}\right) \sin \pi}{\sin \left(\frac{\pi}{n+1}\right)}-\cos \pi+\frac{1}{\left(-2 \cos \left(\frac{\pi}{n+1}\right)\right)^{n+1}} \\
& =1+\frac{1}{\left(-2 \cos \left(\frac{\pi}{n+1}\right)\right)^{n+1}} \\
& >0,
\end{aligned}
$$

since $\left|\frac{1}{\left(-2 \cos \left(\frac{\pi}{n+1}\right)\right)^{n+1}}\right|<1$. Since $g_{K_{n}}$ is continuous and the sign changes from $\theta=0$ to $\theta=\frac{\pi}{n+1}$, we have by the Intermediate Value Theorem that there is at least one zero of $g_{K_{n}}$ on the interval $\left(0, \frac{\pi}{n+1}\right)$. By Lemma 18, $\theta$ is a zero of $K_{n}\left(z_{K}(\theta)\right)$ if and only if $g_{K_{n}}(\theta)=0$, so since $g_{K_{n}}$ is continuous on $(0, \pi / 3)$, the Intermediate Value Theorem implies that there are $\lfloor n / 3\rfloor$ solutions of $K_{n}(z)=0$. Since $\operatorname{deg}\left(K_{n}(z)\right)$ is at most $\lfloor n / 3\rfloor$, these are the only zeros of $K_{n}(z)$ by the Fundamental Theorem of Algebra. Since $z_{K}(\theta):(0, \pi / 3) \rightarrow(-\infty,-4 / 27)$ is a continuous bijective map, we get that all 
of the zeros of $K_{n}(z)$ lie on the real interval $(-\infty,-4 / 27)$, and are dense there as $n \rightarrow \infty$ by Theorem 4 . 


\section{MAIN RESULT}

We now provide necessary and sufficient conditions for the hyperbolicty of $P_{n}(z)$, as well as an explicit interval where the zeros lie, for the case where $A(z)=a z, B(z)=b$, and $C(z)=c z$, where $a, b$, and $c$ are real numbers. If $c=0$ and $a$ and $b$ are nonzero, the denominator becomes a quadratic, which was studied in [9]. If $b=0$ and $a$ and $c$ are nonzero, the denominator becomes a three-term cubic, which was also studied in [9]. If $a=0$ and $b$ and $c$ are nonzero, the denominator becomes a different type of three-term cubic, which was studied in [11]. We will show later that if $b<0$ then $P_{n}(z)$ is not hyperbolic for all large $n$ (c.f. Proposition 40). The main result of this thesis is the following theorem.

Theorem 20. The zeros of the sequence $\left\{P_{n}(z)\right\}_{n=0}^{\infty}$ generated by

$$
\sum_{n=0}^{\infty} P_{n}(z) t^{n}=\frac{1}{1+a z t+b t^{2}+c z t^{3}}, \quad a, b, c \in \mathbb{R} \backslash\{0\},
$$

are real if and only if $b>0$ and $\frac{c}{a b}:=\alpha \leq \frac{1}{9}$, in which case they lie on the interval $(-\lambda, \lambda)$ where

$$
\lambda:=\frac{4}{\left(\frac{3 \alpha+1+\sqrt{9 \alpha^{2}-10 \alpha+1}}{-5 \alpha+1+\sqrt{9 \alpha^{2}-10 \alpha+1}}\right)^{\frac{3}{2}}\left(-5 \alpha+1+\sqrt{9 \alpha^{2}-10 \alpha+1}\right)}
$$

Furthermore, the set of zeros of $P_{n}(z)$ is dense on $(-\lambda, \lambda)$ as $n \rightarrow \infty$. 
To simplify the proof, we make the substitution $t \rightarrow \frac{t}{\sqrt{b}}$, and the generating relation becomes

$$
\sum_{n=0}^{\infty} P_{n}(z)\left(\frac{t}{\sqrt{b}}\right)^{n}=\frac{1}{1+\frac{a}{\sqrt{b}} z t+t^{2}+\frac{c}{b \sqrt{b}} z t^{3}} .
$$

We next make the substitution $\frac{a}{\sqrt{b}} z \rightarrow z^{\prime}$, and the generating relation becomes

$$
\sum_{n=0}^{\infty} P_{n}\left(\frac{\sqrt{b}}{a} z^{\prime}\right)\left(\frac{t}{\sqrt{b}}\right)^{n}=\frac{1}{1+z^{\prime} t+t^{2}+\frac{c}{a b} z^{\prime} t^{3}} .
$$

Since these substitutions only cause scaling and stretching of $P_{n}(z)$, they do not affect the reality (or nonreality) of the zeros, so if we finally make the substitution $\frac{c}{a b} \rightarrow \alpha$, the theorem can be restated in the following equivalent form.

Theorem 21. The zeros of the sequence $\left\{P_{n}(z)\right\}_{n=0}^{\infty}$ generated by

$$
\sum_{n=0}^{\infty} P_{n}(z) t^{n}=\frac{1}{1+z t+t^{2}+\alpha z t^{3}}, \quad \alpha \in \mathbb{R} \backslash\{0\}
$$

are real if and only if $\alpha \leq \frac{1}{9}$, in which case they lie on the interval $(-\lambda, \lambda)$ where

$$
\lambda:=\frac{4}{\left(\frac{3 \alpha+1+\sqrt{9 \alpha^{2}-10 \alpha+1}}{-5 \alpha+1+\sqrt{9 \alpha^{2}-10 \alpha+1}}\right)^{\frac{3}{2}}\left(-5 \alpha+1+\sqrt{9 \alpha^{2}-10 \alpha+1}\right)}
$$

Furthermore, the set of zeros of $P_{n}(z)$ is dense on $(-\lambda, \lambda)$ as $n \rightarrow \infty$.

To prove this theorem, we must establish several lemmas. We begin by defining a few terms. 
Definition 22. For $\theta \in(0, \pi)$, define the functions

$$
\begin{aligned}
t_{P_{1}}(\theta) & :=\tau_{P}(\theta) e^{-i \theta}, \\
t_{P_{2}}(\theta) & :=\tau_{P}(\theta) e^{i \theta}, \\
t_{P_{3}}(\theta) & :=\tau_{P}(\theta) \zeta_{P}(\theta), \\
z_{P}(\theta) & :=\frac{-1}{\alpha \tau_{P}^{3}(\theta) \zeta_{P}(\theta)}, \\
\Delta(\theta) & :=16 \alpha^{2} \cos ^{4} \theta-8 \alpha^{2} \cos ^{2} \theta-8 \alpha \cos ^{2} \theta+\alpha^{2}-2 \alpha+1,
\end{aligned}
$$

where

$$
\begin{aligned}
\tau_{P}(\theta) & :=\sqrt{\frac{2 \cos \theta+\zeta_{P}(\theta)}{\zeta_{P}(\theta)}} \quad \text { and } \\
\zeta_{P}(\theta) & :=\frac{-4 \alpha \cos ^{2} \theta-\alpha+1+\sqrt{\Delta(\theta)}}{4 \alpha \cos \theta} .
\end{aligned}
$$

The following three lemmas establish the fact that $\tau_{P}, \zeta_{P}$, and $z_{P}$ are well-defined.

Lemma 23. For $0 \neq \alpha \leq \frac{1}{9}$ and $\theta \in(0, \pi), \Delta(\theta)>0$.

Proof. First suppose $\alpha=1 / 9$. Then we have

$$
\begin{aligned}
\Delta(\theta) & =\frac{16}{81} \cos ^{4} \theta-\frac{8}{81} \cos ^{2} \theta-\frac{8}{9} \cos ^{2} \theta+\frac{1}{81}-\frac{2}{9}+1 \\
& =\frac{16}{81}\left(\cos ^{4} \theta-5 \cos ^{2} \theta+4\right)
\end{aligned}
$$

which has zeros at $\theta=0$ and $\theta=\pi$, and none in between. Since $\pi / 2 \in(0, \pi)$ and

$$
\Delta\left(\frac{\pi}{2}\right)=\frac{16}{81}\left(\cos ^{4}\left(\frac{\pi}{2}\right)-5 \cos ^{2}\left(\frac{\pi}{2}\right)+4\right)=\frac{64}{81}>0
$$


and since $\Delta(\theta)$ is continuous on $\mathbb{R}$, we have that $\Delta(\theta)>0$ for $\alpha=1 / 9$.

Now suppose $\alpha<1 / 9$ and $\alpha \neq 0$. We find the relative extrema of $\Delta(\theta)$ by differentiating with respect to $\theta$.

$$
\Delta^{\prime}(\theta)=-64 \alpha^{2} \cos ^{3} \theta \sin \theta+16 \alpha^{2} \cos \theta \sin \theta+16 \alpha \cos \theta \sin \theta .
$$

Setting this expression equal to zero gives the equation

$$
-16 \alpha \sin \theta \cos \theta\left(4 \alpha \cos ^{2} \theta-\alpha-1\right)=0 .
$$

Note that $\sin \theta \cos \theta=0$ if $\theta=0, \pi / 2$, and $\pi$, and

$$
4 \alpha \cos ^{2} \theta-\alpha-1=0
$$

when

$$
\theta=\cos ^{-1}\left(\sqrt{\frac{\alpha+1}{4 \alpha}}\right) \text { and } \theta=\cos ^{-1}\left(-\sqrt{\frac{\alpha+1}{4 \alpha}}\right) .
$$

There are two conditions that must be met for the latter solutions to exist. First, $\frac{\alpha+1}{4 \alpha}$ must be greater than or equal to zero, which is not the case for $\alpha \in(-1,0]$. Furthermore, for $\pm \sqrt{\frac{\alpha+1}{4 \alpha}}$ to be in the domain of the inverse cosine function, we must have

$$
\left| \pm \sqrt{\frac{\alpha+1}{4 \alpha}}\right| \leq 1
$$

or equivalently,

$$
\alpha \in(-\infty, 0) \cup[1 / 3, \infty)
$$


Since we are assuming that $\alpha<1 / 9$, we deduce that the inverse cosine solutions only exist for $\alpha \leq-1$.

Observe

$$
\Delta(0)=\Delta(\pi)=9 \alpha^{2}-10 \alpha+1=(9 \alpha-1)(\alpha-1),
$$

which is positive for all $\alpha<1 / 9$. We also have

$$
\Delta(\pi / 2)=\alpha^{2}-2 \alpha+1=(\alpha-1)^{2},
$$

which is positive for all $\alpha \neq 1$. Furthermore,

$$
\begin{aligned}
\Delta\left(\cos ^{-1}\left( \pm \sqrt{\frac{\alpha+1}{4 \alpha}}\right)\right) & =(\alpha+1)^{2}-2 \alpha(\alpha+1)-2(\alpha+1)+\alpha^{2}-2 \alpha+1 \\
& =-4 \alpha
\end{aligned}
$$

which is positive for all $\alpha \leq-1$.

Since all relative extrema on $(0, \pi)$ give positive values of $\Delta(\theta)$, and $\Delta(\theta)$ is continuous on $\mathbb{R}$, we have that $\Delta(\theta)>0$ for $\theta \in(0, \pi)$.

Lemma 24. For $\theta \in(0, \pi)$, let $\zeta_{P}$ be as in Definition 22 with $4 \alpha \cos \theta \neq 0$. Then $\left|\zeta_{P}\right|>1$.

Proof. When deriving the formula for $\zeta_{P}$ we used combinations of $t_{P_{1}}, t_{P_{2}}$, and $t_{P_{3}}$ with Vieta's formulas to obtain the function

$$
f(\zeta):=2 \alpha \cos \theta \zeta^{2}+\left(4 \alpha \cos ^{2} \theta+\alpha-1\right) \zeta+2 \alpha \cos \theta,
$$


which has zeros

$$
\begin{aligned}
& \zeta_{+}:=\zeta_{P}=\frac{-4 \alpha \cos ^{2} \theta-\alpha+1+\sqrt{\Delta(\theta)}}{4 \alpha \cos \theta} \text { and } \\
& \zeta_{-}:=\frac{-4 \alpha \cos ^{2} \theta-\alpha+1-\sqrt{\Delta(\theta)}}{4 \alpha \cos \theta} .
\end{aligned}
$$

Observe that

$$
\begin{aligned}
f(-1)(f(1)= & \left(2 \alpha \cos \theta-4 \alpha \cos ^{2} \theta-\alpha+1+2 \alpha \cos \theta\right) \times \\
& \left(2 \alpha \cos \theta+4 \alpha \cos ^{2} \theta+\alpha-1+2 \alpha \cos \theta\right) \\
= & -16 \alpha^{2} \cos ^{4} \theta+8 \alpha^{2} \cos ^{2} \theta+8 \alpha \cos ^{2} \theta-\alpha^{2}+2 \alpha-1 \\
= & -\Delta(\theta),
\end{aligned}
$$

which is negative for $\theta \in(0, \pi)$ be Lemma 23 . Hence, by the Intermediate Value Theorem, exactly one of the zeros of $f(\zeta)$ lies outside the interval $[-1,1]$

$$
\begin{aligned}
& \text { If } \alpha<0 \text {, then }-4 \alpha \cos ^{2} \theta-\alpha+1>0 \Longrightarrow\left|\zeta_{+}\right|>\left|\zeta_{-}\right| \text {. } \\
& \text { If } 0<\alpha \leq 1 / 9 \text {, then } \\
& \qquad-4 \alpha \cos ^{2} \theta-\alpha+1 \geq-5 \alpha+1>0,
\end{aligned}
$$

and applying the triangle inequality to the numerators, we have

$$
\left|-4 \alpha \cos ^{2} \theta-\alpha+1-\sqrt{\Delta(\theta)}\right| \leq-4 \alpha \cos ^{2} \theta-\alpha+1+\sqrt{\Delta(\theta)},
$$


which again implies that $\left|\zeta_{+}\right|>\left|\zeta_{-}\right|$. Hence we see that $\zeta_{+}=\zeta_{P}$ must lie outside the interval $[-1,1]$ for all $\theta \in(0, \pi)$, and thus $\left|\zeta_{P}\right|>1 \forall \theta \in(0, \pi)$.

Lemma 25. For $0 \neq \alpha \leq 1 / 9$ and $\theta \in(0, \pi), \frac{2 \cos \theta+\zeta_{P}}{\zeta_{P}}>0$.

Proof. Case 1: Suppose $0<\alpha \leq 1 / 9$. Note that

$$
\frac{2 \cos \theta+\zeta_{P}}{\zeta_{P}}=\frac{4 \alpha \cos ^{2} \theta-\alpha+1+\sqrt{\Delta(\theta)}}{-4 \alpha \cos ^{2} \theta-\alpha+1+\sqrt{\Delta(\theta)}}
$$

We show that both the numerator, denoted $N(\theta)$, and denominator, denoted $D(\theta)$, are positive for all $0<\alpha \leq 1 / 9$. Note that the smallest $D(\theta)$ can be is when $\alpha=1 / 9$ and $\cos ^{2} \theta=1$. In that case,

$$
D(\theta)=-4\left(\frac{1}{9}\right)(1)-\frac{1}{9}+1+\sqrt{\Delta(\theta)}=\frac{4}{9}+\sqrt{\Delta(\theta)}>0
$$

by Lemma 23 . Since $4 \cos ^{2} \theta-1<0$ on $(\pi / 3,2 \pi / 3)$, the smallest it can be on that interval is when $\alpha=1 / 9$ and $4 \cos ^{2} \theta-1=-1$. In that case,

$$
N(\theta)=\frac{1}{9}(-1)+1+\sqrt{\Delta(\theta)}=\frac{8}{9}+\sqrt{\Delta(\theta)}>0
$$

by Lemma 23. Since $4 \cos ^{2} \theta-1>0$ on $(0, \pi / 3) \cup(2 \pi / 3, \pi)$, we get $N(\theta)=\alpha\left(4 \cos ^{2} \theta-1\right)+1+\sqrt{\Delta(\theta)}>0$ by Lemma 23.

Case 2: Suppose $\alpha \leq 0$. We show that $\frac{2 \cos \theta}{\zeta_{P}}+1>0$ by showing that $\left|\zeta_{P}\right|>|2 \cos \theta|$ using a similar argument as in Lemma 24. From (16) in Lemma 24, we have

$$
f(-2 \cos \theta) f(2 \cos \theta)=4 \cos ^{2} \theta\left(8 \alpha \cos ^{2} \theta+2 \alpha-1\right)<0
$$


since $\cos ^{2} \theta>0$ and $\alpha<0$. Hence, by the Intermediate Value Theorem, exactly one of the zeros of $f(\zeta)$ lies outside the interval $[-2 \cos \theta, 2 \cos \theta]$, and since $\left|\zeta_{+}\right|>\left|\zeta_{-}\right|$by the proof of Lemma 24, we get that $\left|\zeta_{P}\right|>|2 \cos \theta|$.

Next we show that $t_{P_{1}}, t_{P_{2}}$, and $t_{P_{3}}$ are the zeros of the denominator of the generating function $1+z_{P} t+t^{2}+\alpha z_{P}^{3}$.

Lemma 26. For $z_{P} \neq 0$ and $\alpha \neq 0, t_{P_{1}}, t_{P_{2}}$, and $t_{P_{3}}$ are the zeros of

$$
1+z_{P} t+t^{2}+\alpha z_{P} t^{3}
$$

Proof. We show that $t_{P_{1}}, t_{P_{2}}$, and $t_{P_{3}}$ satisfy Vieta's formulas for $1+z_{P} t+t^{2}+\alpha z_{P} t^{3}$. More precisely, we show that

$$
\begin{aligned}
t_{P_{1}}+t_{P_{2}}+t_{P_{3}} & =-\frac{1}{\alpha z_{P}}, \\
t_{P_{1}} t_{P_{2}}+t_{P_{1}} t_{P_{3}}+t_{P_{2}} t_{P_{3}} & =\frac{1}{\alpha} \\
t_{P_{1}} t_{P_{2}} t_{P_{3}} & =-\frac{1}{\alpha z_{P}} .
\end{aligned}
$$

Note that when we substitute the expressions for $\tau_{P}$ and $\zeta_{P}$ into the expression for $z_{P}$, we have

$$
z_{P}=\frac{4 \cos \theta}{\left(\frac{4 \alpha \cos ^{2} \theta-\alpha+1+\sqrt{\Delta}}{-4 \alpha \cos ^{2} \theta-\alpha+1+\sqrt{\Delta}}\right)^{\frac{3}{2}}\left(4 \alpha \cos ^{2} \theta+\alpha-1-\sqrt{\Delta}\right)},
$$


which is well-defined by Lemmas 23 and 25. Observe

$$
\begin{aligned}
& t_{P_{1}}+t_{P_{2}}+t_{P_{3}}=\tau_{P} e^{-i \theta}+\tau_{P} e^{i \theta}+\tau_{P} \zeta_{P} \\
& =\tau_{P}\left(2 \cos \theta+\zeta_{P}\right) \\
& =\sqrt{\frac{2 \cos \theta+\zeta_{P}}{\zeta_{P}}}\left(2 \cos \theta+\zeta_{P}\right) \\
& =\left(\frac{\left(2 \cos \theta+\zeta_{P}\right)^{3}}{\zeta_{P}}\right)^{\frac{1}{2}} \\
& =\left(\frac{\left(2 \cos \theta+\frac{-4 \alpha \cos ^{2} \theta-\alpha+1+\sqrt{\Delta}}{4 \alpha \cos \theta}\right)^{3}}{\frac{-4 \alpha \cos ^{2} \theta-\alpha+1+\sqrt{\Delta}}{4 \alpha \cos \theta}}\right)^{\frac{1}{2}} \\
& =\left(\frac{1}{4 \alpha \cos \theta}\right)\left(\frac{\left(4 \alpha \cos ^{2} \theta-\alpha+1+\sqrt{\Delta}\right)^{3}}{-4 \alpha \cos ^{2} \theta-\alpha+1+\sqrt{\Delta}}\right)^{\frac{1}{2}} \\
& =\left(\frac{1}{4 \alpha \cos \theta}\right)\left(\frac{\left(4 \alpha \cos ^{2} \theta-\alpha+1+\sqrt{\Delta}\right)^{3}}{-4 \alpha \cos ^{2} \theta-\alpha+1+\sqrt{\Delta}}\right)^{\frac{1}{2}} \times \\
& \left(\frac{\left(-4 \alpha \cos ^{2} \theta-\alpha+1+\sqrt{\Delta}\right)^{2}}{\left(-4 \alpha \cos ^{2} \theta-\alpha+1+\sqrt{\Delta}\right)^{2}}\right)^{\frac{1}{2}} \\
& =\left(\frac{1}{4 \alpha \cos \theta}\right)\left(\left(\frac{4 \alpha \cos ^{2} \theta-\alpha+1+\sqrt{\Delta}}{-4 \alpha \cos ^{2} \theta-\alpha+1+\sqrt{\Delta}}\right)^{3}\right)^{\frac{1}{2}} \times \\
& \left(-4 \alpha \cos ^{2} \theta-\alpha+1+\sqrt{\Delta}\right) \\
& =\frac{\left(\frac{4 \alpha \cos ^{2} \theta-\alpha+1+\sqrt{\Delta}}{-4 \alpha \cos ^{2} \theta-\alpha+1+\sqrt{\Delta}}\right)^{\frac{3}{2}}\left(4 \alpha \cos ^{2} \theta+\alpha-1-\sqrt{\Delta}\right)}{-4 \alpha \cos \theta} \\
& =-\frac{1}{\alpha z_{P}} \text {. }
\end{aligned}
$$


Next we have

$$
\begin{aligned}
t_{P_{1}} t_{P_{2}}+t_{P_{1}} t_{P_{3}}+t_{P_{2}} t_{P_{3}} & =\tau_{P} e^{-i \theta} \tau_{P} e^{i \theta}+\tau_{P} e^{-i \theta} \tau_{P} \zeta_{P}+\tau_{P} e^{i \theta} \tau_{P} \zeta_{P} \\
& =\tau_{P}^{2}\left(1+2 \zeta_{P} \cos \theta\right) \\
& =\left(\sqrt{\frac{2 \cos \theta+\zeta_{P}}{\zeta_{P}}}\right)^{2}\left(1+2 \zeta_{P} \cos \theta\right) \\
& =\left(\frac{2 \cos \theta+\frac{-4 \alpha \cos ^{2} \theta-\alpha+1+\sqrt{\Delta}}{4 \alpha \cos \theta}}{\frac{-4 \alpha \cos ^{2} \theta-\alpha+1+\sqrt{\Delta}}{4 \alpha \cos \theta}}\right) \times \\
& \left(\frac{\left.1+2 \cos \theta\left(\frac{-4 \alpha \cos ^{2} \theta-\alpha+1+\sqrt{\Delta}}{4 \alpha \cos \theta}\right)\right)}{-4 \alpha \cos ^{2} \theta-\alpha+1+\sqrt{\Delta}}\right) \times \\
& \left(\frac{-4 \alpha \cos ^{2} \theta+\alpha+1+\sqrt{\Delta}}{2 \alpha}\right) \\
& =\left(\frac{2\left(-4 \alpha \cos ^{2} \theta-\alpha+1+\sqrt{\Delta}\right)}{-4 \alpha \cos ^{2} \theta-\alpha+1+\sqrt{\Delta}}\right)\left(\frac{1}{2 \alpha}\right) \\
& =\frac{1}{\alpha} \cdot
\end{aligned}
$$


Finally,

$$
\begin{aligned}
t_{P_{1}} t_{P_{2}} t_{P_{3}} & =\tau_{P} e^{-i \theta} \tau_{P} e^{i \theta} \tau_{P} \zeta_{P} \\
& =\tau_{P}^{3} \zeta_{P} \\
& =\left(\sqrt{\frac{2 \cos \theta_{P}+\zeta_{P}}{\zeta_{P}}}\right)^{3} \zeta_{P} \\
& =\left(\frac{2 \cos \theta+\frac{-4 \alpha \cos ^{2} \theta-\alpha+1+\sqrt{\Delta}}{4 \alpha \cos \theta}}{\frac{-4 \alpha \cos ^{2} \theta-\alpha+1+\sqrt{\Delta}}{4 \alpha \cos \theta}}\right)^{\frac{3}{2}}\left(\frac{-4 \alpha \cos ^{2} \theta-\alpha+1+\sqrt{\Delta}}{4 \alpha \cos \theta}\right) \\
& =\left(\frac{4 \alpha \cos ^{2} \theta-\alpha+1+\sqrt{\Delta}}{-4 \alpha \cos ^{2} \theta-\alpha+1+\sqrt{\Delta}}\right)^{\frac{3}{2}}\left(\frac{-4 \alpha \cos ^{2} \theta-\alpha+1+\sqrt{\Delta}}{4 \alpha \cos \theta}\right) \\
& =\frac{\left(\frac{4 \alpha \cos ^{2} \theta-\alpha+1+\sqrt{\Delta}}{-4 \alpha \cos ^{2} \theta-\alpha+1+\sqrt{\Delta}}\right)^{\frac{3}{2}}\left(4 \alpha \cos ^{2} \theta+\alpha-1-\sqrt{\Delta}\right)}{-4 \alpha \cos \theta} \\
& =-\frac{1}{\alpha z_{P}} .
\end{aligned}
$$

Hence since $t_{P_{1}}, t_{P_{2}}$, and $t_{P_{3}}$ satisfy Vieta's formulas, they are the zeros of

$$
1+z_{P} t+t^{2}+\alpha z_{P} t^{3}
$$

Lemma 27. The function $z_{P}$ in Definition 22 is monotone increasing on $(0, \pi)$

Proof. Since $t_{P_{1}}$ is a zero of $1+z_{P} t+t^{2}+\alpha z_{P} t^{3}$,

$$
z_{P}=-\frac{t_{P_{1}}^{2}+1}{t_{P_{1}}+\alpha t_{P_{1}}^{3}}
$$


First suppose that $\alpha<0$. Then we have

$$
z_{P}=-\frac{\left(t_{P_{1}}+i\right)\left(t_{P_{1}}-i\right)}{t_{P_{1}}\left(\sqrt{|\alpha|} t_{P_{1}}+1\right)\left(\sqrt{|\alpha|} t_{P_{1}}-1\right)} .
$$

Taking the derivative by the product rule and then dividing by $z_{P}$ implies that

$$
\frac{d z_{P}}{z_{P}}=\frac{d t_{P_{1}}}{t_{P_{1}}+i}+\frac{d t_{P_{1}}}{t_{P_{1}}-i}-\frac{d t_{P_{1}}}{t_{P_{1}}}-\frac{\sqrt{|\alpha|} d t_{P_{1}}}{\sqrt{|\alpha|} t_{P_{1}}+1}-\frac{\sqrt{|\alpha|} d t_{P_{1}}}{\sqrt{|\alpha|} t_{P_{1}}-1} .
$$

Note that

$$
d t_{P_{1}}=d\left(\tau_{P} e^{-i \theta}\right)=-i \tau_{P} e^{-i \theta} d \theta+e^{-i \theta} d \tau_{P}=t_{P_{1}}\left(\frac{d \tau_{P}}{\tau_{P}}-i d \theta\right)
$$

so if we let

$$
h\left(t_{P_{1}}\right):=\frac{t_{P_{1}}}{t_{P_{1}}+i}+\frac{t_{P_{1}}}{t_{P_{1}}-i}-\frac{t_{P_{1}} \sqrt{|\alpha|}}{\sqrt{|\alpha|} t_{P_{1}}+1}-\frac{t_{P_{1}} \sqrt{|\alpha|}}{\sqrt{|\alpha|} t_{P_{1}}-1}-1,
$$

we have

$$
\frac{d z_{P}}{z_{P}}=h\left(t_{P_{1}}\right)\left(\frac{d \tau_{P}}{\tau_{P}}-i d \theta\right)
$$

or equivalently,

$$
\frac{d z_{P}}{z_{P} d \theta}=h\left(t_{P_{1}}\right)\left(\frac{d \tau_{P}}{\tau_{P} d \theta}-i\right)
$$

Since $\frac{d z_{P}}{z_{P} d \theta} \in \mathbb{R}$ for each $\theta \in(0, \pi)$, we have

$$
0=\operatorname{Im} \frac{d z_{P}}{z_{P} d \theta}=\operatorname{Im}\left(h\left(t_{P_{1}}\right) \frac{d \tau_{P}}{\tau_{P} d \theta}-h\left(t_{P_{1}}\right) i\right)=-\operatorname{Re} h\left(t_{P_{1}}\right)+\operatorname{Im} h\left(t_{P_{1}}\right) \frac{d \tau_{P}}{\tau_{P} d \theta},
$$


which implies

$$
\operatorname{Im} h\left(t_{P_{1}}\right) \frac{d \tau_{P}}{\tau_{P} d \theta}=\operatorname{Re} h\left(t_{P_{1}}\right)
$$

and

$$
\frac{d z_{P}}{z_{P} d \theta}=\operatorname{Re} h\left(t_{P_{1}}\right) \frac{d \tau_{P}}{\tau_{P} d \theta}+\operatorname{Im} h\left(t_{P_{1}}\right)
$$

If we multiply both sides of Equation (17) by $\operatorname{Im} h\left(t_{P_{1}}\right)$, we have

$$
\begin{aligned}
& \operatorname{Im} h\left(t_{P_{1}}\right) \frac{d z_{P}}{z_{P} d \theta}=\operatorname{Im} h\left(t_{P_{1}}\right) \frac{d \tau_{P}}{\tau_{P} d \theta} \operatorname{Re} h\left(t_{P_{1}}\right)+\operatorname{Im} h\left(t_{P_{1}}\right) \operatorname{Im} h\left(t_{P_{1}}\right) \\
\Longrightarrow & \operatorname{Im} h\left(t_{P_{1}}\right) \frac{d z_{P}}{z_{P} d \theta}=\operatorname{Re} h\left(t_{P_{1}}\right) \operatorname{Re} h\left(t_{P_{1}}\right)+\operatorname{Im} h\left(t_{P_{1}}\right) \operatorname{Im} h\left(t_{P_{1}}\right) \\
\Longrightarrow & \operatorname{Im} h\left(t_{P_{1}}\right) \frac{d z_{P}}{d \theta}=z_{P}\left(\operatorname{Re} h\left(t_{P_{1}}\right)\right)^{2}+z_{P}\left(\operatorname{Im} h\left(t_{P_{1}}\right)\right)^{2} \\
\Longrightarrow & \operatorname{Im} h\left(t_{P_{1}}\right) \frac{d z_{P}}{d \theta}=z_{P}\left|h\left(t_{P_{1}}\right)\right|^{2} \\
\Longrightarrow & \frac{d z_{P}}{d \theta}=\frac{z_{P}\left|h\left(t_{P_{1}}\right)\right|^{2}}{\operatorname{Im} h\left(t_{P_{1}}\right)} .
\end{aligned}
$$

Since $\left|h\left(t_{P_{1}}\right)\right|^{2}>0$, the sign of $\frac{d z_{P}}{d \theta}$ depends on $z_{P}$ and $\operatorname{Im} h\left(t_{P_{1}}\right)$. Note that

(18) $\operatorname{Im} h\left(t_{P_{1}}\right)=\operatorname{Im}\left(\frac{t_{P_{1}}}{t_{P_{1}}+i}+\frac{t_{P_{1}}}{t_{P_{1}}-i}-\frac{t_{P_{1}} \sqrt{|\alpha|}}{\sqrt{|\alpha|} t_{P_{1}}+1}-\frac{t_{P_{1}} \sqrt{|\alpha|}}{\sqrt{|\alpha|} t_{P_{1}}-1}-1\right)$.

Multiplying the top and bottom of each fraction by the conjugate of their respective denominator makes Equation (18) equivalent to

$$
\operatorname{Im}\left(\frac{\left|t_{P_{1}}\right|^{2}-t_{P_{1}} i}{\left|t_{P_{1}}+i\right|^{2}}+\frac{\left|t_{P_{1}}\right|^{2}+t_{P_{1}} i}{\left|t_{P_{1}}-i\right|^{2}}-\frac{|\alpha|\left|t_{P_{1}}\right|^{2}+t_{P_{1}} \sqrt{|\alpha|}}{\left|\sqrt{|\alpha|} t_{P_{1}}+1\right|^{2}}-\frac{|\alpha|\left|t_{P_{1}}\right|^{2}-t_{P_{1}} \sqrt{|\alpha|}}{\left|\sqrt{|\alpha|} t_{P_{1}}-1\right|^{2}}\right) .
$$


Since $\left|t_{P_{1}}\right|^{2} \in \mathbb{R}$ and $|\alpha| \in \mathbb{R}, \operatorname{Im} h\left(t_{P_{1}}\right)$ is equivalent to

$$
\operatorname{Im}\left(-\frac{t_{P_{1}} i}{\left|t_{P_{1}}+i\right|^{2}}+\frac{t_{P_{1}} i}{\left|t_{P_{1}}-i\right|^{2}}-\frac{t_{P_{1}} \sqrt{|\alpha|}}{\left|\sqrt{|\alpha|} t_{P_{1}}+1\right|^{2}}+\frac{t_{P_{1}} \sqrt{|\alpha|}}{\left|\sqrt{|\alpha|} t_{P_{1}}-1\right|^{2}}\right) .
$$

We next substitute the expression in Definition 22 for $t_{P_{1}}$ and find

$$
\begin{aligned}
\operatorname{Im} h\left(t_{P_{1}}\right)= & -\frac{\tau_{P} \cos \theta}{\tau_{P}^{2}-2 \tau_{P} \sin \theta+1}+\frac{\tau_{P} \cos \theta}{\tau_{P}^{2}+2 \tau_{P} \sin \theta+1} \\
& +\frac{\sqrt{|\alpha|} \tau_{P} \sin \theta}{|\alpha| \tau_{P}^{2}+2 \sqrt{|\alpha|} \tau_{P} \cos \theta+1}-\frac{\sqrt{|\alpha|} \tau_{P} \sin \theta}{|\alpha| \tau_{P}^{2}-2 \sqrt{|\alpha|} \tau_{P} \cos \theta+1} .
\end{aligned}
$$

When we combine, the common denominator will be a product of squares of modulus, and will thus be a positive value. Hence we can consider just the numerator, which becomes

$$
\begin{aligned}
& -4 \alpha^{2} \tau_{P}^{6} \sin \theta \cos \theta-16|\alpha| \tau_{P}^{4} \sin \theta \cos \theta+16|\alpha| \tau_{P}^{4} \sin \theta \cos ^{3} \theta \\
& -4 \tau_{P}^{2} \sin \theta \cos \theta-4|\alpha| \tau_{P}^{6} \sin \theta \cos \theta+16|\alpha| \tau_{P}^{4} \sin ^{3} \theta \cos \theta-4|\alpha| \tau_{P}^{2} \sin \theta \cos \theta
\end{aligned}
$$

Applying the trigonometric identities $2 \sin \theta \cos \theta=\sin 2 \theta$, and $\sin ^{2} \theta+\cos ^{2} \theta=1$, we obtain

$$
\operatorname{Im} h\left(t_{P_{1}}\right)=-2 \tau_{P}^{2}\left(\sin 2 \theta+|\alpha| \sin 2 \theta+|\alpha| \tau_{P}^{4} \sin 2 \theta+\alpha^{2} \tau_{P}^{4} \sin 2 \theta\right) .
$$

Since $-2 \tau_{P}^{2}<0$, we analyze

$$
\sin 2 \theta+|\alpha| \sin 2 \theta+|\alpha| \tau_{P}^{4} \sin 2 \theta+\alpha^{2} \tau_{P}^{4} \sin 2 \theta=\sin 2 \theta\left(1+|\alpha|+|\alpha| \tau_{P}^{4}+\alpha^{2} \tau_{P}^{4}\right)
$$

on the intervals $(0, \pi / 2)$ and $(\pi / 2, \pi)$. 
On $(0, \pi / 2), z_{P}<0$ and $\sin 2 \theta>0$, and since $1+|\alpha|+|\alpha| \tau_{P}^{4}+\alpha^{2} \tau_{P}^{4}>0$, we have that $\operatorname{Im} h\left(t_{P_{1}}\right)<0$, which implies that $\frac{d z_{P}}{d \theta}>0$ on $(0, \pi / 2)$.

On $(\pi / 2, \pi), z_{P}>0$ and $\sin 2 \theta<0$, so we get that $\operatorname{Im} h\left(t_{P_{1}}\right)>0$, which implies that $\frac{d z_{P}}{d \theta}>0$ on $(\pi / 2, \pi)$. Thus $\frac{d z_{P}}{d \theta}>0$ on $(0, \pi)$ for $\alpha<0$.

Now suppose $0<\alpha \leq 1 / 9$. Then

$$
z_{P}=-\frac{\left(t_{P_{1}}+i\right)\left(t_{P_{1}}-i\right)}{t_{P_{1}}\left(\sqrt{\alpha} t_{P_{1}}+i\right)\left(\sqrt{\alpha} t_{P_{1}}-i\right)}
$$

and taking the derivative by the product rule and dividing by $z_{P}$ implies that

$$
\frac{d z_{P}}{z_{P}}=\frac{d t_{P_{1}}}{t_{P_{1}}+i}+\frac{d t_{P_{1}}}{t_{P_{1}}-i}-\frac{d t_{P_{1}}}{t_{P_{1}}}-\frac{\sqrt{\alpha} d t_{P_{1}}}{\sqrt{\alpha} t_{P_{1}}+i}-\frac{\sqrt{\alpha} d t_{P_{1}}}{\sqrt{\alpha} t_{P_{1}}-i} .
$$

Using a similar process as above, we obtain

$$
\frac{d z_{P}}{d \theta}=\frac{z_{P}\left|k\left(t_{P_{1}}\right)\right|^{2}}{\operatorname{Im} k\left(t_{P_{1}}\right)}
$$

where

$$
k\left(t_{P_{1}}\right):=\frac{t_{P_{1}}}{t_{P_{1}}+i}+\frac{t_{P_{1}}}{t_{P_{1}}-i}-\frac{t_{P_{1}} \sqrt{\alpha}}{\sqrt{\alpha} t_{P_{1}}+i}-\frac{t_{P_{1}} \sqrt{\alpha}}{\sqrt{\alpha} t_{P_{1}}-i}-1 .
$$

Since $\left|k\left(t_{P_{1}}\right)\right|^{2}>0$, the sign of $\frac{d z_{P}}{d \theta}$ again depends on $z_{P}$ and $\operatorname{Im} k\left(t_{P_{1}}\right)$. We evaluate $\operatorname{Im} k\left(t_{P_{1}}\right)$ in the same way. We have

$$
\operatorname{Im} k\left(t_{P_{1}}\right)=\operatorname{Im}\left(\frac{t_{P_{1}}}{t_{P_{1}}+i}+\frac{t_{P_{1}}}{t_{P_{1}}-i}-\frac{t_{P_{1}} \sqrt{\alpha}}{\sqrt{\alpha} t_{P_{1}}+i}-\frac{t_{P_{1}} \sqrt{\alpha}}{\sqrt{\alpha} t_{P_{1}}-i}-1\right)
$$


Multiplying the top and bottom of each fraction by the conjugate of their respective denominator makes Equation (19) equivalent to

$$
\operatorname{Im}\left(\frac{\left|t_{P_{1}}\right|^{2}-t_{P_{1}} i}{\left|t_{P_{1}}+i\right|^{2}}+\frac{\left|t_{P_{1}}\right|^{2}+t_{P_{1}} i}{\left|t_{P_{1}}-i\right|^{2}}+\frac{t_{P_{1}} \sqrt{\alpha} i-\alpha\left|t_{P_{1}}\right|^{2}}{\left|\sqrt{\alpha} t_{P_{1}}+i\right|^{2}}+\frac{-t_{P_{1}} \sqrt{\alpha} i-\alpha\left|t_{P_{1}}\right|^{2}}{\left|\sqrt{\alpha} t_{P_{1}}-i\right|^{2}}\right) .
$$

Since $\left|t_{P_{1}}\right|^{2} \in \mathbb{R}$ and $\sqrt{\alpha} \in \mathbb{R}, \operatorname{Im} k\left(t_{P_{1}}\right)$ is equivalent to

$$
\operatorname{Im}\left(-\frac{t_{P_{1}} i}{\left|t_{P_{1}}+i\right|^{2}}+\frac{t_{P_{1}} i}{\left|t_{P_{1}}-i\right|^{2}}+\frac{t_{P_{1}} \sqrt{\alpha} i}{\left|\sqrt{\alpha} t_{P_{1}}+i\right|^{2}}-\frac{t_{P_{1}} \sqrt{\alpha} i}{\left|\sqrt{\alpha} t_{P_{1}}-i\right|^{2}}\right) .
$$

We next substitute the expression in Definition 22 for $t_{P_{1}}$ and find

$$
\begin{aligned}
\operatorname{Im} k\left(t_{P_{1}}\right)= & -\frac{\tau_{P} \cos \theta}{\tau_{P}^{2}-2 \tau_{P} \sin \theta+1}+\frac{\tau_{P} \cos \theta}{\tau_{P}^{2}+2 \tau_{P} \sin \theta+1} \\
& +\frac{\sqrt{\alpha} \tau_{P} \cos \theta}{\alpha \tau_{P}^{2}-2 \sqrt{\alpha} \tau_{P} \sin \theta+1}-\frac{\sqrt{\alpha} \cos \theta}{\alpha \tau_{P}^{2}+2 \sqrt{\alpha} \tau_{P} \sin \theta+1}
\end{aligned}
$$

As before, when we combine, the common denominator will be a product of squares of modulus, and will thus be a positive value. Hence we can can consider just the numerator, which becomes

$$
-2 \alpha^{2} \tau_{P}^{6} \sin \theta \cos \theta-4 \tau_{P}^{2} \sin \theta \cos \theta+4 \alpha \tau_{P}^{6} \sin \theta \cos \theta+4 \alpha \tau_{P}^{2} \sin \theta \cos \theta .
$$

Applying the trigonometric identity $2 \sin \theta \cos \theta=\sin 2 \theta$, we obtain

$$
\operatorname{Im} k\left(t_{1}\right)=-2 \tau_{P}^{2}\left(\sin 2 \theta-\alpha \sin 2 \theta-\alpha \tau_{P}^{4} \sin 2 \theta+\alpha^{2} \tau_{P}^{4} \sin 2 \theta\right)
$$


Since $-2 \tau_{P}^{2}<0$, we analyze

$\sin 2 \theta-\alpha \sin 2 \theta-\alpha \tau_{P}^{4} \sin 2 \theta+\alpha^{2} \tau_{P}^{4} \sin 2 \theta=\sin 2 \theta\left(1-\alpha-\alpha \tau_{P}^{4}+\alpha^{2} \tau_{P}^{4}\right)$

on $(0, \pi / 2)$ and $(\pi / 2, \pi)$.

Suppose first that $\theta \in(0, \pi / 2)$. Then $z_{P}<0$ and $\sin 2 \theta>0$. Note that

$$
1-\alpha-\alpha \tau_{P}^{4}+\alpha^{2} \tau_{P}^{4}=(1-\alpha)\left(1-\alpha \tau_{P}^{4}\right)
$$

Since $1-\alpha>0$ for $0<\alpha \leq 1 / 9$, we must show that $1-\alpha \tau_{P}^{4}>0$. Note that $1-\alpha \tau_{P}^{4}$ is smallest when $\alpha=1 / 9$. Note that $\tau_{P}^{4}=\left(\frac{2 \cos \theta}{\zeta_{P}}+1\right)^{2}$, and since $\left|\tau_{P}\right|>1$ by Lemma 24, we have

$$
0<\frac{2 \cos \theta}{\zeta_{P}}+1<3 \Longrightarrow \tau_{P}^{4}<9
$$

Hence $1-\alpha \tau_{P}^{4}>0$, and thus $\frac{d z_{P}}{d \theta}>0$ on $(0, \pi / 2)$.

Now suppose $\theta \in(\pi / 2, \pi)$. Then $z_{P}>0$ and $\sin 2 \theta<0$. With the same argument as above, we get that $1-\alpha \tau_{P}^{4}>0$, implying that $\frac{d z_{P}}{d \theta}>0$ on $(\pi / 2, \pi)$.

Hence $\frac{d z_{P}}{d \theta}>0$ on $(0, \pi)$, and thus $z_{P}$ is increasing on $(0, \pi)$.

Lemma 28. For $\theta \in(0, \pi), z_{P} \in(-\lambda, \lambda)$ where

$$
\lambda:=\frac{4}{\left(\frac{3 \alpha+1+\sqrt{9 \alpha^{2}-10 \alpha+1}}{-5 \alpha+1+\sqrt{9 \alpha^{2}-10 \alpha+1}}\right)^{\frac{3}{2}}\left(-5 \alpha+1+\sqrt{9 \alpha^{2}-10 \alpha+1}\right)}
$$


Proof. By Lemma 27, $z_{P}$ is increasing on $(0, \pi)$, so it suffices to check $\lim _{\theta \rightarrow 0^{+}} z_{P}$ and $\lim _{\theta \rightarrow \pi^{-}} z_{P}$. Observe

$$
\begin{aligned}
\lim _{\theta \rightarrow 0^{+}} z_{P} & =\lim _{\theta \rightarrow 0^{+}} \frac{4 \cos \theta}{\left(\frac{4 \alpha \cos ^{2} \theta-\alpha+1+\sqrt{\Delta}}{-4 \alpha \cos ^{2} \theta-\alpha+1+\sqrt{\Delta}}\right)^{\frac{3}{2}}\left(4 \alpha \cos ^{2} \theta+\alpha-1-\sqrt{\Delta}\right)} \\
& =\frac{4}{\left(\frac{3 \alpha+1+\sqrt{9 \alpha^{2}-10 \alpha+1}}{-5 \alpha+1+\sqrt{9 \alpha^{2}-10 \alpha+1}}\right)^{\frac{3}{2}}\left(5 \alpha-1-\sqrt{9 \alpha^{2}-10 \alpha+1}\right)} \\
& =\frac{-4}{\left(\frac{3 \alpha+1+\sqrt{9 \alpha^{2}-10 \alpha+1}}{-5 \alpha+1+\sqrt{9 \alpha^{2}-10 \alpha+1}}\right)^{\frac{3}{2}}\left(-5 \alpha+1+\sqrt{9 \alpha^{2}-10 \alpha+1}\right)} \\
& =-\lambda .
\end{aligned}
$$

Similarly,

$$
\begin{aligned}
\lim _{\theta \rightarrow \pi^{-}} z_{P} & =\lim _{\theta \rightarrow \pi^{-}} \frac{4 \cos \theta}{\left(\frac{4 \alpha \cos ^{2} \theta-\alpha+1+\sqrt{\Delta}}{-4 \alpha \cos ^{2} \theta-\alpha+1+\sqrt{\Delta}}\right)^{\frac{3}{2}}\left(4 \alpha \cos ^{2} \theta+\alpha-1-\sqrt{\Delta}\right)} \\
& =\frac{-4}{\left(\frac{3 \alpha+1+\sqrt{9 \alpha^{2}-10 \alpha+1}}{-5 \alpha+1+\sqrt{9 \alpha^{2}-10 \alpha+1}}\right)^{\frac{3}{2}}\left(5 \alpha-1-\sqrt{9 \alpha^{2}-10 \alpha+1}\right)} \\
& =\frac{4}{\left(\frac{3 \alpha+1+\sqrt{9 \alpha^{2}-10 \alpha+1}}{-5 \alpha+1+\sqrt{9 \alpha^{2}-10 \alpha+1}}\right)^{\frac{3}{2}}\left(-5 \alpha+1+\sqrt{9 \alpha^{2}-10 \alpha+1}\right)} \\
& =\lambda .
\end{aligned}
$$

Lemma 29. For each $n \in \mathbb{N}$, the degree of $P_{n}(z)$ is $n$.

Proof. Note that $\left\{P_{n}(z)\right\}_{n=0}^{\infty}$ satisfies the recurrence

$$
P_{n}(z)=-z P_{n-1}(z)-P_{n-2}(z)-\alpha z P_{n-3}(z), n \geq 3,
$$


where

$$
\begin{aligned}
& P_{0}(z)=1 \\
& P_{1}(z)=-z \\
& P_{2}(z)=z^{2}-1 .
\end{aligned}
$$

We induct on $n$ to show that $\operatorname{deg}\left(P_{n}(z)\right)=n$.

In the case where $n=0, P_{0}(z)=1$, which has degree 0 . Suppose now that $\operatorname{deg}\left(P_{n}(z)\right)=n$. Then

$$
\begin{aligned}
\operatorname{deg}\left(P_{n+1}(z)\right) & =\operatorname{deg}\left(-z P_{n}(z)-P_{n-1}(z)-\alpha z P_{n-2}(z)\right) \\
& =\operatorname{deg}\left(P_{n}(z)\right)+1 \\
& =n+1
\end{aligned}
$$

Hence by induction we see that $\operatorname{deg}\left(P_{n}(z)\right)=n$ for all $n$.

Using the function $\zeta_{P}$ in Definition 22, define

$$
g_{P_{n}}(\theta):=\frac{\left(\zeta_{P}-\cos \theta\right) \sin (n+1) \theta}{\sin \theta}-\cos (n+1) \theta+\frac{1}{\zeta_{P}^{n+1}}
$$

By Lemma 18, $\theta$ is a zero of $P_{n}\left(z_{P}(\theta)\right)$ if and only if $g_{P_{n}}(\theta)=0$. Note that when $\cos (n+1) \theta= \pm 1, \sin (n+1) \theta=0$. By Lemma 24, we know that $\left|\zeta_{P}\right|>1$, so we see that when $\cos (n+1) \theta=1, g_{P_{n}}<0$, and when $\cos (n+1) \theta=-1, g_{P_{n}}>0$.

Hence, by the Intermediate Value Theorem, the function $g_{P_{n}}$ has at least one zero on each subinterval whose endpoints are the solutions of 
$\cos (n+1) \theta= \pm 1$. However, $g_{P_{n}}$ has a vertical asymptote in the subinterval containing $\theta=\pi / 2$ because $\zeta_{P}$ has a vertical asymptote at $\theta=\pi / 2$. Note that on $(0, \pi)$,

$$
\begin{aligned}
& \cos (n+1) \theta= \pm 1 \\
\Longrightarrow & \theta=\frac{k \pi}{n+1}, k \in \mathbb{Z}, \\
\Longrightarrow & k \in\{1,2,3, \ldots, n\},
\end{aligned}
$$

which means there are $n-1$ subintervals on $(0, \pi)$, excluding the endpoints at 0 and $\pi$, and as stated above each subinterval besides the one containing the vertical asymptote has at least one zero of $g_{P_{n}}$. So we have found $n-2$ zeros of $g_{P_{n}}$ on $(0, \pi)$. Since we have shown in Lemma 29 that the degree of $P_{n}(z)$ is $n$, we must find the missing zeros. Since there is a vertical asymptote at $\theta=\pi / 2$, it is possible that the subinterval containing $\pi / 2$ has multiple zeros or no zeros, with the latter situation meaning there are two missing zeros. The following lemmas settle these cases. We will need to consider the cases where $\alpha<0$ and $0<\alpha \leq 1 / 9$ separately. Note that the interval $(0, \pi)$ is partitioned into $n+1$ subintervals with endpoints

$$
0, \frac{\pi}{n+1}, \frac{2 \pi}{n+1}, \frac{3 \pi}{n+1}, \ldots, \frac{n \pi}{n+1}, \pi
$$

Lemma 30. For $\alpha<0$, the function $g_{P_{n}}$ defined in Equation (20) has at least one zero on each of the intervals

$$
\left(0, \frac{\pi}{n+1}\right) \text { and }\left(\frac{n \pi}{n+1}, \pi\right) \text {. }
$$


Proof. Recall that

$$
g_{P_{n}}=\frac{\left(\zeta_{P}-\cos \theta\right) \sin (n+1) \theta}{\sin \theta}-\cos (n+1) \theta+\frac{1}{\zeta_{P}^{n+1}}
$$

and the term $-\cos (n+1) \theta$ determines the sign of $g_{P_{n}}(\theta)$ at each interval endpoint.

$$
\begin{aligned}
& \text { For } \theta=\frac{\pi}{n+1} \text {, we have } \\
& \qquad \begin{aligned}
\\
\qquad \begin{array}{l}
\cos (n+1) \theta=-\cos \pi=-(-1)=1,
\end{array}
\end{aligned}
\end{aligned}
$$

which implies that $g_{P_{n}}>0$ at $\frac{\pi}{n+1} \forall n \in \mathbb{N}$.

Note that

$$
\lim _{\theta \rightarrow 0^{+}}\left(\zeta_{P}-\cos \theta\right)<0,
$$

and

$$
\lim _{\theta \rightarrow 0^{+}} \frac{\sin (n+1) \theta}{\sin \theta}=n+1
$$

Furthermore, since $\cos (0)=1$ and $\left|\frac{1}{\zeta_{P}^{n+1}}\right|<1$, we have

$$
\lim _{\theta \rightarrow 0^{+}} g_{P_{n}}<0 \forall n \in \mathbb{N}
$$

Since $g_{P_{n}}$ is continuous on $\left(0, \frac{\pi}{n+1}\right)$ and it changes sign from 0 to $\frac{\pi}{n+1}$, we have by the Intermediate Value Theorem that $g_{P_{n}}$ has at least one zero on $\left(0, \frac{\pi}{n+1}\right)$.

For the interval $\left(\frac{n \pi}{n+1}, \pi\right)$, we must consider the two cases when $n$ is even and when $n$ is odd. 
First, suppose $n$ is even. So $n=2 \ell$ for some $\ell \in \mathbb{N}$.

For $\theta=\frac{n \pi}{n+1}$, we have

$$
-\cos (n+1) \theta=-\cos (2 \ell \pi)=-1,
$$

which implies $g_{P_{n}}<0$ at $\frac{n \pi}{n+1} \forall n \in 2 \mathbb{N}$.

Note that

$$
\lim _{\theta \rightarrow \pi^{-}}\left(\zeta_{P}-\cos \theta\right)>0
$$

and

$$
\lim _{\theta \rightarrow \pi^{-}} \frac{\sin (n+1) \theta}{\sin \theta}=\lim _{\theta \rightarrow \pi^{-}} \frac{\sin (2 \ell \theta+\theta)}{\sin \theta}=\lim _{\theta \rightarrow \pi^{-}} \frac{(2 \ell+1) \cos (2 \ell \theta+\theta)}{\cos \theta}=2 \ell+1 .
$$

Furthermore, since

$$
-\cos (2 \ell+1) \pi=-\cos (2 \ell \pi+\pi)=-(-1)=1,
$$

and

$$
\left|\frac{1}{\zeta_{P}^{n+1}}\right|<1
$$

we ascertain that

$$
\lim _{\theta \rightarrow \pi^{-}} g_{P_{n}}>0 \forall n \in 2 \mathbb{N} .
$$

Since $g_{P_{n}}$ is continuous on $\left(\frac{n \pi}{n+1}, \pi\right)$ and it changes sign from $\frac{n \pi}{n+1}$ to $\pi$, the Intermediate Value Theorem implies that $g_{P_{n}}$ has at least one zero on $\left(\frac{n \pi}{n+1}, \pi\right)$ if $n$ is even.

Now suppose that $n$ is odd and write $n=2 \ell+1$ for some $\ell \in \mathbb{N}$. 
For $\theta=\frac{n \pi}{n+1}$, we have

$$
-\cos (n+1) \theta=-\cos (2 \ell \pi+\pi)=-(-1)=1,
$$

which implies that $g_{P_{n}}>0$ at $\frac{n \pi}{n+1}=\frac{(2 \ell+1) \pi}{2 \ell+2} \forall \ell \in \mathbb{N}$.

As before,

$$
\lim _{\theta \rightarrow \pi^{-}}\left(\zeta_{P}-\cos \theta\right)>0
$$

but

$$
\begin{aligned}
\lim _{\theta \rightarrow \pi^{-}} \frac{\sin (n+1) \theta}{\sin \theta} & =\lim _{\theta \rightarrow \pi^{-}} \frac{\sin (2 \ell \theta+2 \theta)}{\sin \theta} \\
& =\lim _{\theta \rightarrow \pi^{-}} \frac{(2 \ell+2) \cos (2 \ell \theta+2 \theta)}{\cos \theta} \\
& =-(2 \ell+2) .
\end{aligned}
$$

Furthermore, since

$$
-\cos (2 \ell+2) \pi=-1
$$

and

$$
\left|\frac{1}{\zeta_{P}^{n+1}}\right|<1
$$

we have

$$
\lim _{\theta \rightarrow \pi^{-}} g_{P_{n}}<0 \forall \ell \in \mathbb{N} .
$$

Since $g_{P_{n}}$ is continuous on $\left(\frac{n \pi}{n+1}, \pi\right)$ and it changes sign from $\frac{n \pi}{n+1}$ to $\pi$, the Intermediate Value Theorem implies that $g_{P_{n}}$ has at least one zero on $\left(\frac{n \pi}{n+1}, \pi\right)$ for $n$ odd. 
In the next lemma, we will use the fact that for $0<\alpha \leq 1 / 9$,

$$
\lim _{\theta \rightarrow \frac{\pi}{2}^{-}}\left(\zeta_{P}-\cos \theta\right)=+\infty
$$

$$
\lim _{\theta \rightarrow \frac{\pi}{2}^{+}}\left(\zeta_{P}-\cos \theta\right)=-\infty
$$

and the terms $-\cos (n+1) \theta$ and $\frac{1}{\zeta_{P}^{n+1}}$ are finite at $\theta=\pi / 2$. Note that if $n$ is even, then the vertical asymptote at $\theta=\frac{\pi}{2}$ lies in the center of the central subinterval

$$
\left(\frac{n \pi}{2 n+2}, \frac{(n+2) \pi}{2 n+2}\right)
$$

Lemma 31. For $0<\alpha \leq 1 / 9$ and $n$ even, $g_{P_{n}}$ has at least one zero on the intervals

$$
\left(\frac{n \pi}{2 n+2}, \frac{\pi}{2}\right) \text { and }\left(\frac{\pi}{2}, \frac{(n+2) \pi}{2 n+2}\right)
$$

Proof. We must consider the case where $n$ is a multiple of 4 and when $n$ is not a multiple of 4 separately.

First, suppose $n$ is a multiple of 4 . Then $n=4 \ell$ for some $\ell \in \mathbb{N}$. So

$$
\lim _{\theta \rightarrow \frac{\pi}{2} \pm} \frac{\sin (n+1) \theta}{\sin \theta}=\sin (2 \ell \theta+\pi / 2)=1 .
$$

Hence Equations (21) and (22) together with Equation (23) imply that

$$
\lim _{\theta \rightarrow \frac{\pi}{2}^{-}} g_{P_{n}}(\theta)=+\infty
$$


and

$$
\lim _{\theta \rightarrow \frac{\pi}{2}^{+}} g_{P_{n}}(\theta)=-\infty
$$

Next we determine the sign of $g_{P_{n}}$ at the endpoints $\frac{n \pi}{2 n+2}$ and $\frac{(n+2) \pi}{2 n+2}$ by finding the sign of $-\cos (n+1) \theta$. Observe that for $n=4 \ell$ and $\theta=\frac{n \pi}{2 n+2}$,

$$
-\cos (n+1) \theta=-\cos (2 \ell \theta)=-1 \text {. }
$$

For $n=4 \ell$ and $\theta=\frac{(n+2) \pi}{2 n+2}$,

$$
-\cos (n+1) \theta=-\cos (2 \ell \pi+\pi)=1 \text {. }
$$

Equations (24) and (26) imply that $g_{P_{n}}$ changes sign on the interval $\left(\frac{n \pi}{2 n+2}, \frac{\pi}{2}\right)$, and Equations (25) and (27) imply that $g_{P_{n}}$ changes sign on the interval $\left(\frac{\pi}{2}, \frac{(n+2) \pi}{2 n+2}\right)$. Since $g_{P_{n}}$ is continuous on those intervals, we have by the Intermediate Value Theorem that $g_{P_{n}}$ has at least one zero on each of those intervals for $n=4 \ell$.

Now suppose $n$ is not a multiple of 4 . So $n=4 \ell-2$ for some $\ell \in \mathbb{N}$. So

$$
\lim _{\theta \rightarrow \frac{\pi}{2} \pm} \frac{\sin (n+1) \theta}{\sin \theta}=\sin (2 \ell \pi-\pi / 2)=-1 .
$$

Hence Equations (21) and (22) together with Equation (28) imply that

$$
\lim _{\theta \rightarrow \frac{\pi}{2}^{-}} g_{P_{n}}(\theta)=-\infty
$$


and

$$
\lim _{\theta \rightarrow \frac{\pi}{2}^{+}} g_{P_{n}}(\theta)=+\infty
$$

Again, we determine the sign of $g_{P_{n}}$ at the endpoints by finding the sign of $-\cos (n+1) \theta$. Observe that for $n=4 \ell-2$ and $\theta=\frac{n \pi}{2 n+2}$,

$$
-\cos (n+1) \theta)=-\cos (2 \ell \pi-\pi)=1
$$

For $n=4 \ell-2$ and $\theta=\frac{(n+2) \pi)}{2 n+2}$,

$$
-\cos (n+1) \theta=-\cos (2 \ell \pi)=-1 \text {. }
$$

Equations (29) and (31) imply that $g_{P_{n}}$ changes sign on the interval $\left(\frac{n \pi}{2 n+2}, \frac{\pi}{2}\right)$, and Equations (30) and (32) imply that $g_{P_{n}}$ changes sign on the interval $\left(\frac{\pi}{2}, \frac{(n+2) \pi}{2 n+2}\right)$. Since $g_{P_{n}}$ is continuous on those intervals, we again have by the Intermediate Value Theorem that $g_{P_{n}}$ has at least one zero on each of those intervals for $n=4 \ell-2$.

Note that if $n$ is odd, then the possible discontinuity at $\theta=\frac{\pi}{2}$ occurs directly on the central subinterval endpoint.

Lemma 32. For $0<\alpha \leq 1 / 9$ and $n$ odd, $g_{P_{n}}$ has at least one zero on the intervals

$$
\left(\frac{(n-1) \pi}{2 n+2}, \frac{\pi}{2}\right) \text { and }\left(\frac{\pi}{2}, \frac{(n+3) \pi}{2 n+2}\right) \text {. }
$$


Proof. Let $n$ be an odd natural number. We must consider the case where $n=4 \ell-1$ and $n=4 \ell+1, \ell \in \mathbb{N}$, separately.

First, suppose $n=4 \ell-1$. Then since $0<\alpha \leq 1 / 9$,

$$
\lim _{\theta \rightarrow \frac{\pi}{2}} \frac{\left(\zeta_{P}-\cos \theta\right) \sin (n+1) \theta}{\sin \theta}=\frac{1-\alpha}{\alpha}(-2 \ell) .
$$

Since $|\cos (n+1) \theta| \leq 1$ and $\left|\frac{1}{\zeta_{P}}\right|<1$, Equation (33) implies that

$$
\lim _{\theta \rightarrow \frac{\pi}{2}} g_{P_{n}}(\theta)<0
$$

Next we determine the sign of $g_{P_{n}}$ at the endpoints $\frac{(n-1) \pi}{2 n+2}$ and $\frac{(n+3) \pi}{2 n+2}$ by finding the sign of $-\cos (n+1) \theta$. Observe that for $n=4 \ell-1$ and $\theta=\frac{(n-1) \pi}{2 n+2}$

$$
-\cos (n+1) \theta=-\cos (2 \ell \pi-\pi)=1 \text {. }
$$

For $n=4 \ell-1$ and $\theta=\frac{(n+3) \pi}{2 n+2}$,

$$
-\cos (n+1) \theta=-\cos (2 \ell \pi+\pi)=1 \text {. }
$$

Equations (34) and (35) imply that $g_{P_{n}}$ changes sign on the interval $\left(\frac{(n-1) \pi}{2 n+2}, \frac{\pi}{2}\right)$, and Equations (34) and (36) imply that $g_{P_{n}}$ changes sign on the interval $\left(\frac{\pi}{2}, \frac{(n+3) \pi}{2 n+2}\right)$. Since $g_{P_{n}}$ is continuous on those intervals, we have by the Intermediate Value Theorem that $g_{P_{n}}$ has at least one zero on each of those intervals for $n=4 \ell-1$. 
Now suppose $n=4 \ell+1$ for some $\ell \in \mathbb{N}$. Then since $0<\alpha \leq 1 / 9$,

$$
\lim _{\theta \rightarrow \frac{\pi}{2}} \frac{\left(\zeta_{P}-\cos \theta\right) \sin (n+1) \theta}{\sin \theta}=\frac{1-\alpha}{\alpha}(2 \ell+1) .
$$

Since $|\cos (n+1) \theta| \leq 1$ and $\left|\frac{1}{\zeta_{P}}\right|<1$, Equation (37) implies that

$$
\lim _{\theta \rightarrow \frac{\pi}{2}} g_{P_{n}}(\theta)>0
$$

We again determine the sign of $g_{P_{n}}$ at the endpoints $\frac{(n-1) \pi}{2 n+2}$ and $\frac{(n+3) \pi}{2 n+2}$ by finding the sign of $-\cos (n+1) \theta$. Observe that for $n=4 \ell+1$ and $\theta=\frac{(n-1) \pi}{2 n+2}$

$$
-\cos (n+1) \theta=-\cos (2 \ell \pi)=-1 \text {. }
$$

For $n=4 \ell+1$ and $\theta=\frac{(n+3) \pi}{2 n+2}$,

$$
-\cos (n+1) \theta=-\cos (2 \ell \pi+2 \pi)=-1 .
$$

Equations (38) and (39) imply that $g_{P_{n}}$ changes sign on the interval $\left(\frac{(n-1) \pi}{2 n+2}, \frac{\pi}{2}\right)$, and Equations (38) and (40) imply that $g_{P_{n}}$ changes sign on the interval $\left(\frac{\pi}{2}, \frac{(n+3) \pi}{2 n+2}\right)$. Since $g_{P_{n}}$ is continuous on those intervals, we again have by the Intermediate Value Theorem that $g_{P_{n}}$ has at least one zero on each of those intervals for $n=4 \ell+1$.

By Lemmas 30,31 , and 32 , we see that $g_{P_{n}}$ has a total of $n$ zeros on the interval $(0, \pi)$ when $n$ is even, and a total of $n-1$ zeros on the interval 
$(0, \pi)$ when $n$ is odd. By Lemma 29 , the degree of $P_{n}(z)$ is $n$ for all $n \in \mathbb{N}$, so the Fundamental Theorem of Algebra tells us that there must be exactly one more zero when $n$ is odd.

Lemma 33. If $n$ is odd, then $z=0$ is a zero of $P_{n}(z)$.

Proof. Recall that the generating relation for $\left\{P_{n}(z)\right\}_{n=0}^{\infty}$ is

$$
\sum_{n=0}^{\infty} P_{n}(z) t^{n}=\frac{1}{1+z t+t^{2}+\alpha z t^{3}} .
$$

Then the generating relation for $z=0$ becomes

$$
\sum_{n=0}^{\infty} P_{n}(0) t^{n}=\frac{1}{1+t^{2}}
$$

Expanding both sides gives

$$
P_{0}(0)+P_{1}(0) t+P_{2}(0) t^{2}+P_{3}(0) t^{3}+P_{4}(0) t^{4}+\cdots=1-t^{2}+t^{4}-t^{6}+t^{8}-\ldots
$$

Equating coefficients yields

$$
P_{n}(0)=\left\{\begin{array}{cc}
0 & \text { if } n \text { is odd } \\
\pm 1 & \text { if } n \text { is even }
\end{array}\right.
$$

Hence $P_{n}(0)=0$ for all odd $n$.

By Lemma 18, we conclude that since $g_{P_{n}}$ has $n$ zeros on $(0, \pi)$ for all $n \in \mathbb{N}, P_{n}(z)$ has $n$ zeros on the interval $(-\lambda, \lambda)$ via the monotone map $z_{P}(\theta):(0, \pi) \rightarrow(-\lambda, \lambda)$. Since the degree of $P_{n}(z)$ is $n$ for each $n$, it follows 
by the Fundamental Theorem of Algebra that all of the zeros of $P_{n}(z)$ are real and lie on the interval $(-\lambda, \lambda)$.

Lemma 34. The set of zeros of $P_{n}(z)$ is dense on the open interval $(-\lambda, \lambda)$ as $n \rightarrow \infty$.

Proof. Let $\mathcal{Z}\left(g_{P_{n}}\right)=\left\{\theta \in(0, \pi) \mid g_{n}(\theta)=0\right\}$. Since the solutions of $\cos (n+1) \theta= \pm 1$ are dense on $(0, \pi)$ as $n \rightarrow \infty$, it is clear that $\bigcup_{n=0}^{\infty} \mathcal{Z}\left(g_{P_{n}}\right)$ is dense on $(0, \pi)$. Since $z_{P}:(0, \pi) \rightarrow(-\lambda, \lambda)$ is a continuous bijective function, it follows from Theorem 4 that $\bigcup_{n=0}^{\infty} \mathcal{Z}\left(P_{n}\right)$ is dense on $(-\lambda, \lambda)$.

This concludes the sufficiency part of the proof, where $\alpha \leq 1 / 9$ implies that $P_{n}(z)$ is hyperbolic for all $n$. In the next section, we focus on the necessary part of the proof, where $\alpha>1 / 9$ or $b<0$ implies that $P_{n}(z)$ is not hyperbolic for all large $n$. 


\section{NECESSARY CONDITION FOR THE REALITY OF ZEROS}

To prove that $P_{n}(z)$ is not hyperbolic for large $n$ when $b<0$ or $\alpha>1 / 9$, we will employ the Implicit Function Theorem and a theorem by Sokal in [8], but we must first define a few terms to make sense of the statement of Sokal's theorem.

Definition 35. Let

$$
f_{n}(z)=\sum_{k=1}^{m} \alpha_{k}(z) \beta_{k}(z)^{n}, n \in \mathbb{N}
$$

be a function where $\alpha_{k}(z)$ and $\beta_{k}(z)$ are analytic in a domain $D$. We say that an index $k$ is dominant at $z$ if $\left|\beta_{k}(z)\right| \geq\left|\beta_{l}(z)\right|$ for all $1 \leq l \leq m$.

Definition 36. Let

$$
D_{k}:=\{z \in D \mid k \text { is dominant at } z\} .
$$

We say that $\underline{\lim } \mathcal{Z}\left(f_{n}\right)$ is the set of all $z \in D$ such that every neighborhood $U$ of $z$ has a nonempty intersection with all but finitely many of the sets $\mathcal{Z}\left(f_{n}\right)$, and $\varlimsup \mathcal{Z} \mathcal{Z}\left(f_{n}\right)$ is the set of all $z \in D$ such that every neighborhood $U$ of $z$ has a nonempty intersection with infinitely many of the sets $\mathcal{Z}\left(f_{n}\right)$.

In other words, Definition 36 says that $z \in \underline{\lim } \mathcal{Z}\left(f_{n}\right)$ if for any neighborhood $U$ of $z, f_{n}$ has a zero in $U$ for all $n$ large enough, and $z \in \varlimsup \overline{\lim } \mathcal{Z}\left(f_{n}\right)$ if for any neighborhood $U$ of $z$, infinitely many $f_{n}$ have a zero in $U$. 
Theorem 37. (Sokal [8]) Let $D$ be a domain in $\mathbb{C}$ and let $\alpha_{1}, \ldots, \alpha_{m}, \beta_{1}, \ldots, \beta_{m}(m \geq 2)$ be analytic functions on $D$, none of which is identically zero. Let us further assume a "no degenerate dominance" condition: there do not exist indices $k \neq k^{\prime}$ such that $\beta_{k} \equiv \omega \beta_{k^{\prime}}$ for some constant $\omega$ with $|\omega|=1$ and such that $D_{k}\left(=D_{k^{\prime}}\right)$ has nonempty interior. For each integer $n \geq 0$, define $f_{n}$ by

$$
f_{n}(z)=\sum_{k=1}^{m} \alpha_{k}(z) \beta_{k}(z)^{n}
$$

Then $\underline{\lim } \mathcal{Z}\left(f_{n}\right)=\varlimsup \mathcal{I} \mathcal{Z}\left(f_{n}\right)$, and a point $z^{*}$ lies in this set if and only if either

1. there is a unique dominant index $k$ at $z^{*}$, and $\alpha_{k}\left(z^{*}\right)=0$, or

2. there are two or more dominant indices at $z^{*}$.

Example 38. Consider the function

$$
\frac{1}{(t-i z)(t+z)(t+2 z)} \text {. }
$$

Then

$$
\begin{aligned}
& \beta_{1}(z)=\frac{1}{i z} \\
& \beta_{2}(z)=\frac{1}{-z} \\
& \beta_{3}(z)=\frac{1}{-2 z} .
\end{aligned}
$$


So

$$
\begin{aligned}
& D_{1}=\left\{z \in D|| \beta_{1}(z)|\geq| \beta_{l}(z) \mid \text { for } 1 \leq l \leq 3\right\}=D=\mathbb{C}, \\
& D_{2}=\left\{z \in D|| \beta_{2}(z)|\leq| \beta_{l}(z) \mid \text { for } 1 \leq l \leq 3\right\}=D=\mathbb{C}, \\
& D_{3}=\left\{z \in D|| \beta_{3}(z)|\leq| \beta_{l}(z) \mid \text { for } 1 \leq l \leq 3\right\} \neq D .
\end{aligned}
$$

Note that the "no degenerate dominance" part of Theorem 37 is not satisfied, since $\left|\beta_{1}(z)\right|=\left|\beta_{2}(z)\right|$ and $\mathbb{C}$ does not have empty interior.

We will also apply the Implicit Function Theorem, which we state as the following theorem.

Theorem 39. (Implicit Function Theorem [5]) Let $f_{j}(w, z), j=1, \ldots, m$, be analytic functions of $(w, z)=\left(w_{1}, \ldots, w_{m}, z_{1}, \ldots, z_{n}\right)$ in a neighborhood of a point $\left(w^{*}, z^{*}\right)$ in $\mathbb{C}^{m} \times \mathbb{C}^{n}$, and assume that $f_{j}\left(w^{*}, z^{*}\right)=0, j=1, \ldots, m$, and that

$$
\operatorname{det}\left(\frac{\partial f_{j}}{\partial w_{k}}\right)_{j, k=1}^{m} \neq 0 \text { at }\left(w^{*}, z^{*}\right) .
$$

Then the equations $f_{j}(w, z)=0, j=1, \ldots, m$, have a uniquely determined analytic solution $w(z)$ in a neighborhood of $z^{*}$ such that $w\left(z^{*}\right)=w^{*}$.

We will later find a $z^{*} \in \mathbb{C} \backslash \mathbb{R}$ such that the zeros $t_{1}^{*}, t_{2}^{*}$, and $t_{3}^{*}$ of $1+z^{*} t+t^{2}+\alpha z^{*} t^{3}$ satisfy

$$
\left|t_{1}^{*}\right|=\left|t_{2}^{*}\right| \leq\left|t_{3}^{*}\right|,
$$


where $t_{1}^{*}, t_{2}^{*}$, and $t_{3}^{*}$ are distinct and nonzero. Assuming that we can find such a $z^{*}$, we now claim that $z^{*} \in \underline{\lim } \mathcal{Z}\left(P_{n}\right)$, which implies that $P_{n}(z)$ is not hyperbolic for all large $n$.

First of all, Theorem 39 implies that for a fixed $z^{*} \in \mathbb{C} \backslash \mathbb{R}$, there exist domains $D_{1}, D_{2}$, and $D_{3}$ containing $z^{*}$ and analytic functions $t_{P_{1}}(z), t_{P_{2}}(z)$, and $t_{P_{3}}(z)$ such that

$$
\begin{aligned}
& D\left(t_{P_{1}}(z), z\right)=0 \forall z \in D_{1}, \\
& D\left(t_{P_{2}}(z), z\right)=0 \forall z \in D_{2}, \\
& D\left(t_{P_{3}}(z), z\right)=0 \forall z \in D_{3},
\end{aligned}
$$

where $D(t, z)$ is the bivariate denominator of the generating function of $P_{n}(z)$. Let $D=\bigcap_{k=1}^{3} D_{k}$. We supress the parameter $z$ and note that by continuity, $t_{P_{1}} \neq t_{P_{2}} \neq t_{P_{3}} \neq 0 \forall z \in D$. Note that $P_{n}(z)$ is of the form of Equation (41) since Lemma 13 gives

$$
\begin{aligned}
P_{n}(z)= & -\frac{1}{\left(t_{P_{1}}-t_{P_{2}}\right)\left(t_{P_{1}}-t_{P_{3}}\right) t_{P_{1}}^{n+1}} \\
& -\frac{1}{\left(t_{P_{2}}-t_{P_{1}}\right)\left(t_{P_{2}}-t_{P_{3}}\right) t_{P_{2}}^{n+1}} \\
& -\frac{1}{\left(t_{P_{3}}-t_{P_{1}}\right)\left(t_{P_{3}}-t_{P_{2}}\right) t_{P_{3}}^{n+1}}
\end{aligned}
$$


where

$$
\begin{aligned}
& \alpha_{1}(z)=-\frac{1}{\left(t_{P_{1}}-t_{P_{2}}\right)\left(t_{P_{1}}-t_{P_{3}}\right) t_{P_{1}}}, \\
& \alpha_{2}(z)=-\frac{1}{\left(t_{P_{2}}-t_{P_{1}}\right)\left(t_{P_{2}}-t_{P_{3}}\right) t_{P_{2}}}, \\
& \alpha_{3}(z)=-\frac{1}{\left(t_{P_{3}}-t_{P_{1}}\right)\left(t_{P_{3}}-t_{P_{2}}\right) t_{P_{3}}}
\end{aligned}
$$

and

$$
\begin{aligned}
& \beta_{1}(z)=\frac{1}{t_{P_{1}}}, \\
& \beta_{2}(z)=\frac{1}{t_{P_{2}}}, \\
& \beta_{3}(z)=\frac{1}{t_{P_{3}}},
\end{aligned}
$$

all of which are analytic on $D$ since $t_{P_{1}} \neq t_{P_{2}} \neq t_{P_{3}} \neq 0 \forall z \in D$. For the "no degenerate dominance" condition of Theorem 37, for a fixed $\omega$ on the unit circle, we will show that the set of $z \in D$ such that $\beta_{1}(z)=\omega \beta_{2}(z)$ has empty interior. Let $\omega \in \mathbb{C}$ where $|\omega|=1$ such that $\beta_{1}(z) \equiv \omega \beta_{2}(z) \forall z \in D$. Then $\omega=e^{2 i \theta}$ for some fixed $\theta$ and $t_{P_{1}}=\omega t_{P_{2}}$, which means $t_{P_{1}}=\tau e^{-i \theta}, t_{P_{2}}=\tau e^{i \theta}$, and $t_{P_{3}}=\tau \zeta$ for some $\tau, \zeta \in \mathbb{C} \backslash\{0\}$. By Vieta's formulas, we know

$$
\begin{aligned}
t_{P_{1}}+t_{P_{2}}+t_{P_{3}} & =-\frac{1}{\alpha z}, \\
t_{P_{1}} t_{P_{2}}+t_{P_{1}} t_{P_{3}}+t_{P_{2}} t_{P_{3}} & =\frac{1}{\alpha}, \quad \text { and } \\
t_{P_{1}} t_{P_{2}} t_{P_{3}} & =-\frac{1}{\alpha z} .
\end{aligned}
$$


Substituting $t_{P_{1}}=\tau e^{-i \theta}, t_{P_{2}}=\tau e^{i \theta}$, and $t_{P_{3}}=\tau \zeta$ into Equations (42) and (44), we obtain

$$
z=-\frac{1}{\alpha \tau^{3} \zeta}
$$

and

$$
\tau(2 \cos \theta+\zeta)=\tau^{3} \zeta
$$

which, since $\tau \neq 0$, is equivalent to

$$
\tau^{2}=\frac{2 \cos \theta+\zeta}{\zeta}
$$

Substituting $t_{P_{1}}=\tau e^{-i \theta}, t_{P_{2}}=\tau e^{i \theta}$, and $t_{P_{3}}=\tau \zeta$ into Equation (43), we have

$$
\tau^{2}(1+2 \zeta \cos \theta)=\frac{1}{\alpha}
$$

Substituting the expression for $\tau^{2}$ from Equation (46) into Equation (47) gives

$$
\frac{2 \cos \theta+\zeta}{\zeta}(1+2 \zeta \cos \theta)=\frac{1}{\alpha}
$$

or equivalently,

$$
2 \alpha \cos \theta \zeta^{2}+\left(4 \alpha \cos ^{2} \theta+\alpha-1\right) \zeta+2 \alpha \cos \theta=0
$$

Since $\theta$ is fixed, the sets of $\tau$ and $\zeta$ satisfying Equations (47) and (48) are finite, and hence the set of $z$ satisfying Equation (45) is also finite, and a finite set has empty interior. If $z^{*} \in \mathbb{C} \backslash \mathbb{R}$ such that the zeros in $t$ of 
$1+z^{*} t+t^{2}+\alpha z^{*} t^{3}$ are distinct and nonzero on $D$, then by Lemma 13 and Theorem 37, $z^{*} \in \underline{\lim } \mathcal{Z}\left(P_{n}\right)$ when the two smallest (in modulus) zeros have the same modulus. This is because $\left|t_{P_{1}}\left(z^{*}\right)\right|=\left|t_{P_{2}}\left(z^{*}\right)\right|$, and the second condition of Theorem 37 is satisfied at $z^{*}$ since

$\left|\beta_{1}\left(z^{*}\right)\right|=\left|\beta_{2}\left(z^{*}\right)\right| \geq\left|\beta_{3}\left(z^{*}\right)\right|$ is equivalent to $\left|t_{P_{1}}\left(z^{*}\right)\right|=\left|t_{P_{2}}\left(z^{*}\right)\right| \leq\left|t_{P_{3}}\left(z^{*}\right)\right|$.

The following proposition proves that $P_{n}(z)$ is not hyperbolic for all large $n$ if $b<0$.

Proposition 40. For the sequence $\left\{P_{n}(z)\right\}_{n=0}^{\infty}$ generated by

$$
\sum_{n=0}^{\infty} P_{n}(z) t^{n}=\frac{1}{1+z t-t^{2}+c z t^{3}}, \quad c \in \mathbb{R}
$$

the polynomials $P_{n}(z)$ are not hyperbolic for all large $n \in \mathbb{N}$.

Proof. Case 1: $c=0$. Then the generating relation reduces to

$$
\sum_{n=0}^{\infty} P_{n}(z) t^{n}=\frac{1}{1+z t-t^{2}}
$$

By [10] the zeros of this sequence lie on the curve defined by

$$
\operatorname{Im} \frac{z^{2}}{-1}=0 \text { and } 0 \leq \operatorname{Re} \frac{z^{2}}{-1} \leq 4
$$

In particular, the zeros lie on the curve defined by $z^{2} \in \mathbb{R}$ and $-4 \leq z^{2} \leq 0$, which implies that the zeros lie on the imaginary interval $(-2 i, 2 i)$. 
Case 2: $c \neq 0$. Define

$$
D(t):=1+z t-t^{2}+c z t^{3}
$$

with zeros $t_{1}, t_{2}$, and $t_{3}$, and consider the reciprocal polynomial

$$
D^{*}(t):=t^{3}+z t^{2}-t+c z
$$

Let the zeros of $D^{*}(t)$ be $t_{1}^{*}, t_{2}^{*}$, and $t_{3}^{*}$. We will show that there exists a $z^{*} \in \mathbb{C} \backslash \mathbb{R}$ such that $\left|t_{1}^{*}\right|=\left|t_{2}^{*}\right| \geq\left|t_{3}^{*}\right|$.

Let $\epsilon \in \mathbb{R} \backslash\{0\}$ and let $z^{*}=i \epsilon$. Note that if $z=0$ then the zeros of $D^{*}(t)$ are 0,1 , and -1 . By the continuity of $D^{*}(t)$, it is clear that as $\epsilon \rightarrow 0$,

$$
\begin{aligned}
& t_{1}^{*} \rightarrow 1, \\
& t_{2}^{*} \rightarrow-1, \quad \text { and } \\
& t_{3}^{*} \rightarrow 0 .
\end{aligned}
$$

Note that for our choice of $z^{*}, z^{*}=-\overline{z^{*}}$. Since $t_{1}^{*}$ is a zero of $D^{*}(t)$,

$$
t_{1}^{* 3}+z t_{1}^{* 2}-t_{1}^{*}+c z=0 .
$$


Observe

$$
\begin{aligned}
\left(-\overline{t_{1}^{*}}\right)^{3}+z^{*}\left(-\overline{t_{1}^{*}}\right)^{2}-\left(-\overline{t_{1}^{*}}\right)+c z^{*} & =-\overline{t_{1}^{*}}-\overline{z^{*} \bar{t}_{1}^{2}}+\overline{t_{1}^{*}}-c \overline{z^{*}} \\
& =\overline{-t_{1}^{* 3}-z^{*} t_{1}^{* 2}+t-c z^{*}} \\
& =0 .
\end{aligned}
$$

Hence $-\overline{t_{1}^{*}}:=t_{2}^{*}$ is also a zero of $D^{*}(t)$, and thus

$$
\left|t_{1}^{*}\right|=\left|t_{2}^{*}\right| \geq\left|t_{3}^{*}\right|
$$

for sufficiently small $\epsilon$.

Since the zeros of $D^{*}(t)$ are the reciprocals of the zeros of $D(t)$, we have $\left|t_{1}\right|=\left|t_{2}\right| \leq\left|t_{3}\right|$.

Proposition 41. The zeros of the sequence $\left\{P_{n}(z)\right\}_{n=0}^{\infty}$ generated by

$$
\sum_{n=0}^{\infty} P_{n}(z) t^{n}=\frac{1}{1+z t+t^{2}+\alpha z t^{3}}, \alpha \in \mathbb{R},
$$

are not all real if $\alpha>1 / 9$ for all large $n$.

Proof. Case 1: Suppose $\alpha>1$. Consider $z=i$. Then the denominator of the generating relation becomes

$$
D(t):=1+i t+t^{2}+\alpha i t^{3}
$$


We now show that if $t_{1}, t_{2}$, and $t_{3}$ are the zeros of $D(t)$, then $\left|t_{1}\right|=\left|t_{2}\right| \leq\left|t_{3}\right|$.

Note that $z=i \Longrightarrow z=-\bar{z}$. Suppose $t_{1}$ is a zero of $D(t)$, so that

$$
1+i t_{1}+t_{1}^{2}+\alpha i t_{1}^{3}=0
$$

Then

$$
\begin{aligned}
1+i\left(-\overline{t_{1}}\right)+\left(-\overline{t_{1}}\right)^{2}+\alpha i\left(-\overline{t_{1}}\right)^{3} & =1-i \overline{t_{1}}+{\overline{t_{1}}}^{2}-\alpha i{\overline{t_{1}}}^{3} \\
& =1+\overline{i \bar{t}_{1}}+{\overline{t_{1}}}^{2}+\alpha \overline{i \bar{t}_{1}} \\
& =\overline{1+i t_{1}+t_{1}^{2}+\alpha i t_{1}^{3}} \\
& =0 .
\end{aligned}
$$

Hence $-\overline{t_{1}}:=t_{2}$ is also a zero of $D(t)$. So we have $\left|t_{1}\right|=\left|t_{2}\right|$. It remains to show that $\left|t_{3}\right| \geq\left|t_{1}\right|$.

Let us make the substitution $i t \rightarrow y$ so that we may work with a cubic polynomial with real coefficients. Then our function $D(t)$ becomes

$$
P(y):=1+y-y^{2}-\alpha y^{3} .
$$

Note that the discriminant of a cubic polynomial $a x^{3}+b x^{2}+c x+d$ is

$$
\Delta=18 a b c d-4 b^{3} d+b^{2} c^{2}-4 a c^{3}-27 a^{2} d^{2} .
$$

The discriminant of $P(y)$ is then

$$
\Delta=5+22 \alpha-27 \alpha^{2} .
$$


Since $\alpha>1, \Delta<0$, which implies that $P(y)$ has one real zero and two non-real complex conjugate zeros.

Let $y_{1}, y_{2}$, and $y_{3}$ be the zeros of $P(y)$ with

$$
y_{1}=\tau e^{i \theta}, y_{2}=\tau e^{-i \theta} \text {, and } y_{3} \text { is real. }
$$

We now show that $\left|y_{3}\right|>\tau$.

By Vieta's formulas, we have

$$
\begin{aligned}
y_{1}+y_{2}+y_{3} & =-\frac{1}{\alpha}, \\
y_{1} y_{2}+y_{1} y_{3}+y_{2} y_{3} & =-\frac{1}{\alpha} \\
y_{1} y_{2} y_{3} & =\frac{1}{\alpha} .
\end{aligned}
$$

The last equality gives

$$
\tau^{2} y_{3}=\frac{1}{\alpha} \Longrightarrow \tau^{2}=\frac{1}{\alpha y_{3}}
$$

Note that $\left|y_{1}\right|=\left|y_{2}\right|=\tau$, so we want to show that $\tau^{2}<y_{3}^{2}$, or equivalently, $y_{3}>\frac{1}{\sqrt[3]{\alpha}}$. Observe

$$
P\left(\frac{1}{\sqrt[3]{\alpha}}\right)=1+\frac{1}{\sqrt[3]{\alpha}}-\frac{1}{\sqrt[3]{\alpha^{2}}}-\alpha\left(\frac{1}{\alpha}\right)=\frac{1}{\sqrt[3]{\alpha}}-\frac{1}{\sqrt[3]{\alpha^{2}}}
$$


which is greater than 0 since $\alpha>1$. Furthermore,

$$
\begin{aligned}
\lim _{y \rightarrow-\infty} P(y) & =+\infty \\
\text { and } \lim _{y \rightarrow \infty} P(y) & =-\infty .
\end{aligned}
$$

Since $P\left(y_{3}\right)=0$ and $P\left(\frac{1}{\sqrt[3]{\alpha}}\right)>0$, and $y_{3}$ is the only real zero of $P(y)$, we get that $y_{3}>\frac{1}{\sqrt[3]{\alpha}}$ by the Intermediate Value Theorem.

Case 2: Suppose $1 / 9<\alpha \leq 1$. Since

$$
\lim _{\theta \rightarrow 0} \Delta(\theta)=9 \alpha^{2}-11 \alpha+1<0
$$

by the continuity of $\Delta(\theta)$ there is $\theta^{*}$ sufficiently close to 0 so that $\Delta\left(\theta^{*}\right)<0$. For this choice of $\theta^{*}$, we have $\zeta^{*}:=\zeta_{P}\left(\theta^{*}\right) \notin \mathbb{R}$ and hence $\tau^{*}:=\tau_{P}\left(\theta^{*}\right) \notin \mathbb{R}$. It is clear that $\zeta^{*}$ and $\tau^{*}$ are not real, but we must verify that $z^{*}:=z_{P}\left(\theta^{*}\right)$ is not real.

Suppose by way of contradiction that $z^{*} \in \mathbb{R}$. Then

$$
g(t):=1+z^{*} t+t^{2}+\alpha z^{*} t^{3}
$$

is a polynomial in $t$ with real coefficients.

By Lemma 26,

$$
\begin{aligned}
& t_{1}^{*}:=\tau^{*} e^{-i \theta}, \\
& t_{2}^{*}:=\tau^{*} e^{i \theta}, \\
& t_{3}^{*}:=\tau^{*} \zeta^{*}
\end{aligned}
$$


are the zeros of $g(t)$. Since $g(t)$ is a polynomial with real coefficients, we know complex zeros occur in conjugate pairs. So

$$
\left|t_{1}^{*}\right|=\left|t_{2}^{*}\right| \Longrightarrow \tau^{*} \in \mathbb{R},
$$

a contradiction. Hence $z^{*} \notin \mathbb{R}$. Since $\left|t_{3}^{*}\right|>1$ by Lemma 24 , we have

$$
\left|t_{1}^{*}\right|=\left|t_{2}^{*}\right| \leq\left|t_{3}^{*}\right|,
$$

and hence we have by Theorem 37 that $P_{n}(z)$ is not hyperbolic for large $n$.

Thus, we have shown that if $b<0$ or $\alpha>1 / 9, P_{n}(z)$ is not hyperbolic for all large $n$. 


\section{CONCLUSIONS}

We began this work by studying a famous orthogonal sequence of polynomials called the Chebyshev polynomials of the second kind, denoted $\left\{U_{n}(z)\right\}_{n=0}^{\infty}$. Since this sequence is orthogonal, it can be defined by a three-term recurrence, and each polynomial in the sequence is hyperbolic. We generalized the generating relation for the Chebyshev polynomials to form the sequence $\left\{H_{n}(z)\right\}_{n=0}^{\infty}$, which can be used to find the curve where the zeros lie for any three-term recurrence (whose generating function has quadratic denominator in $t$ ). Next we proved the hyperbolicity of the sequence $\left\{K_{n}(z)\right\}_{n=0}^{\infty}$, which was much more complicated since its generating function has a cubic denominator. The techniques used in the proof were then applied to the main theorem, where the sequence $\left\{P_{n}(z)\right\}_{n=0}^{\infty}$ satisfies a four-term recurrence, and its generating function also has a cubic denominator. Finally, we used a powerful theorem by Sokal to show that $P_{n}(z)$ is not hyperbolic if $b<0$ or if $\alpha>1 / 9$ for all large $n$.

For future work, one may wish to characterize the sequence of polynomials $\left\{Q_{n}(z)\right\}_{n=0}^{\infty}$ defined by

$$
\sum_{n=0}^{\infty} Q_{n}(z) t^{n}=\frac{1}{1+A(z) t+B(z) t^{2}+C(z) t^{3}}
$$


where

$$
\begin{aligned}
& A(z):=a_{1} z+a_{2}, \\
& B(z):=b_{1} z+b_{2}, \quad \text { and } \\
& C(z):=c_{1} z+c_{2}
\end{aligned}
$$

are linear functions with $a_{1}, a_{2}, b_{1}, b_{2}, c_{1}, c_{2} \in \mathbb{R}$. In this thesis, the main result, Theorem 20, characterizes the case where $a_{2}, b_{1}$, and $c_{2}$ are zero. 


\section{REFERENCES}

[1] Abramowitz, Milton; Stegun, Irene A. Handbook of mathematical functions with formulas, graphs, and mathematical tables. National Bureau of Standards Applied Mathematics Series, 55 For sale by the Superintendent of Documents, U.S. Government Printing Office, Washington, D.C. (1964).

[2] Conte, S. D. Elementary numerical analysis: An algorithmic approach. McGraw-Hill Book Co., New York-Toronto, Ont.-London (1965).

[3] Conway, John B. Functions of one complex variable. Second edition. Graduate Texts in Mathematics, 11. Springer-Verlag, New York-Berlin, (1978).

[4] Forgács, Tamás; Tran, Khang. Zeros of polynomials generated by a rational function with a hyperbolic-type denominator. Constr. Approx. 46 (2017), no. 3, 617-643.

[5] Hörmander, Lars. An introduction to complex analysis in several variables. Third edition. North-Holland Mathematical Library, 7. North-Holland Publishing Co., Amsterdam, (1990).

[6] Milovanovi, Gradimir V.(YU-NISEE) Orthogonal polynomial systems and some applications. Inner product spaces and applications, 115182, Pitman Res. Notes Math. Ser., 376, Longman, Harlow, (1997).

[7] Munkres, James R. Topology. Second edition of [MR0464128]. Prentice Hall, Inc., Upper Saddle River, NJ, (2000).

[8] Sokal, Alan D.(1-NY-P) Chromatic roots are dense in the whole complex plane. (English summary) Combin. Probab. Comput. 13 (2004), no. 2, 221-261.

[9] Tran, Khang. Connections between discriminants and the root distribution of polynomials with rational generating function. J. Math. Anal. Appl. 410 (2014), no. 1, 330-340.

[10] Tran, Khang. The root distribution of polynomials with a three-term recurrence. J. Math. Anal. Appl. 421 (2015), no. 1, 878-892.

[11] Tran, Khang; Zumba, Andres. Zeros of polynomials with four-term recurrence. Involve 11 (2018), no. 3, 501-518. 